\title{
Chimeric protein repair of laminin polymerization ameliorates muscular dystrophy phenotype
}

\author{
Karen K. McKee, ${ }^{1}$ Stephanie C. Crosson, ${ }^{1}$ Sarina Meinen, ${ }^{2}$ Judith R. Reinhard, ${ }^{2}$ Markus A. Rüegg, ${ }^{2}$ and Peter D. Yurchenco
}

${ }^{1}$ Robert Wood Johnson Medical School, Rutgers University, Piscataway, New Jersey, USA. ${ }^{2}$ Biozentrum, University of Basel, Basel, Switzerland.

\begin{abstract}
Mutations in laminin $\alpha 2$-subunit (Lma2, encoded by LAMA2) are linked to approximately $30 \%$ of congenital muscular dystrophy cases. Mice with a homozygous mutation in Lama2 (dy2J mice) express a nonpolymerizing form of laminin-211 (Lm211) and are a model for ambulatory-type Lma2-deficient muscular dystrophy. Here, we developed transgenic dy2J mice with muscle-specific expression of $\alpha$ LNNd, a laminin/nidogen chimeric protein that provides a missing polymerization domain. Muscle-specific expression of $\alpha$ LNNd in dy2J mice resulted in strong amelioration of the dystrophic phenotype, manifested by the prevention of fibrosis and restoration of forelimb grip strength. aLNNd also restored myofiber shape, size, and numbers to control levels in dy2J mice. Laminin immunostaining and quantitation of tissue extractions revealed increased Lm211 expression in $\alpha$ LNNd-transgenic dy2J mice. In cultured myotubes, we determined that $\alpha$ LNNd expression increased myotube surface accumulation of polymerization-deficient recombinant laminins, with retention of collagen IV, reiterating the basement membrane (BM) changes observed in vivo. Laminin LN domain mutations linked to several of the Lma2-deficient muscular dystrophies are predicted to compromise polymerization. The data herein support the hypothesis that engineered expression of $\alpha$ LNNd can overcome polymerization deficits to increase laminin, stabilize BM structure, and substantially ameliorate muscular dystrophy.
\end{abstract}

\section{Introduction}

Laminin-211 ( $\alpha 2 \beta 1 \gamma 1$ subunits, abbreviated herein as Lm211) is the major laminin of the basement membrane (BM) of skeletal muscle and peripheral nerve Schwann cells. Mutations within the LAMA2 gene coding for laminin $\alpha 2$ (referred to herein as $L \mathrm{~m} \alpha 2$ ) cause an estimated $30 \%$ of the cases of congenital muscular dystrophy (type MDC1A). These mutations usually result in a complete loss of protein subunit expression. The pathology consists of muscle degeneration and regeneration, chronic inflammation, and fibrosis that can be accompanied by reduced peripheral nerve conduction (1). Patients with mutations resulting in absent or very low protein expression levels fail to ambulate and often die of muscle wasting and respiratory failure by their second decade of life (1). Missense mutations clustered in the Lm $\alpha 2 \mathrm{~N}$-terminal (LN) globular domain $\alpha 2 \mathrm{LN}$, which probably adversely affect polymerization, also occur $(2,3$, and reviewed in ref. 4). Human $\alpha 2 \mathrm{LN}$ domain mutations with retained Lm $\alpha 2$ subunit expression include $\mathrm{Y} 138 \mathrm{H}, \mathrm{Q} 167 \mathrm{P}$, L243P, and G248R, which are located on a polymerization face identified in $\alpha 2 \mathrm{LN}$ domain $(4,5)$. Patients with this type of mutation have little-to-modest reduced protein levels present, with a milder limb-girdle-type dystrophy, in which ambulation is achieved $(3,6,7)$. There is currently no effective treatment for these dystrophies.

\section{Related Commentary: p. 798}

Conflict of interest: The authors have declared that no conflict of interest exists. Submitted: September 23, 2016; Accepted: December 30, 2016 Reference information: J Clin Invest. 2017;127(3):1075-1089. https://doi.org/10.1172/JCI90854.
The sarcolemmal BM can be regarded as a layer of transversely linked extracellular matrix (matrix) proteins extending from stromal collagens to cell-surface receptors to cytoskeleton. This BM also contains laterally interlinked proteins consisting of the laminin and collagen IV polymer networks that stabilize the transverse linkages (8). Two major types of interaction anchor the BM to the muscle cytoskeleton. These interactions take place in a complex of dystroglycan (DG), dystrophin, and dystrophin-associated glycoproteins on the one hand, and integrin $\alpha 7 \beta 1$ and associated cytoplasmic adaptor proteins on the other (9-11). The BM ligands for $\alpha$-dystroglycan $(\alpha D G)$ in skeletal muscle are the C-terminal LG globular domains of Lm211, agrin, and perlecan. Integrin $\alpha 7 \beta 1$ interacts with $\alpha 2$-laminins and, when present, $\alpha 1$ - and $\alpha 5$-laminins (12). DG may provide the more critical link to the cytoskeleton in muscle (13), as a number of mutations found to alter the $\alpha$ DG mannosyl- $O$-linked carbohydrate critical for ligand binding cause severe congenital and limb-girdle dystrophies (1).

A model of BM assembly and structure, based on biochemical, cell, and genetic studies performed by us and others has provided a basis from which to interpret the BM changes that occur in laminin deficiency (14-16). In this model, assembly is initiated by laminins that bind to $\alpha \mathrm{DG}$, integrin, and sulfated glycolipids, the first two providing critical anchorage to the underlying cytoskeleton and the latter increasing the laminin-binding capacity of the cell surface. Nidogen attaches to laminin to form a stable bridge with collagen IV. The proteoglycans agrin and perlecan, the first binding to the laminin coiled-coil and the latter binding to the internally located nidogen globular G2 domain, bestow additional anchorage (collateral linkage) by binding to $\alpha$ DG. For Lm211 (like Lm111), the LG-ligating receptors are $\alpha$ DG and integrin $\alpha 7 \beta 1$. The laminin then polymerizes through interactions of the 3 different short-arm LN domains to form a sheet-like 
laminin network, linking the laminins and their receptor- and matrix-binding partners into a cohesive unit $(5,17-21)$.

Compositional and structural changes underlie the BM alterations in Lm $\alpha 2$ deficiency. In one mouse model of severe loss of Lma2 $\left(d y^{W} / d y^{W}\right.$ mice), the absence of Lm211 results in increased transcription and protein expression of Lm411, with a more limited increase in Lm511 (22). It has been deduced that the compensatory Lm 411 is unable to polymerize because it lacks an $\alpha$-short arm with its critical LN domain (16). Furthermore, there is evidence from protein and protein fragment studies that the LG domains of Lm411 bind poorly to $\alpha$ DG and integrins $(12,23)$. While Lm511 binds to several integrins and can polymerize, its LG domains have been reported to bind less well to integrin $\alpha 7 \beta 1$ and $\alpha \mathrm{DG}(5$, $12,24)$. Thus, one explanation for the severe muscular dystrophy seen in MDC1A patients is a failure of the muscle BM to properly assemble via the LN domains and to be linked to the muscle sarcolemma via the LG domains.

Homozygous $d y^{2 J} / d y^{2 J}$-mutant mice (abbreviated herein as $d y 2 J$ mice) lack $\alpha 2 \mathrm{LN}$ polymerization activity and serve as a model for the ambulatory type of Lm $\alpha 2$ deficiency that is thought to result from a failure of polymerization (25-28). The measured polymerization deficiency of a truncated Lm211 in vitro and the $d y 2 J$ phenotype suggest a significant contribution of the LN domain-mediated laminin network to muscle fiber stability (25). These considerations have led us to evaluate the specifically designed protein called $\alpha \mathrm{LNNd}$, which possesses an $\alpha 1 \mathrm{~N}$-terminal LN polymerization domain and binds to the laminin $\gamma 1$ chain via a nidogen-binding site (15). To this end, we sought to test the laminin assembly model and the validity of the $d y 2 J$ polymerization deficit hypothesis and to explore a possible new therapeutic approach for muscular dystrophy. Initially engineered to provide a gain-of-function tool to probe laminin self-assembly, it was predicted that $\alpha \mathrm{LNNd}$ would also provide the matrix-forming structural linkages lost in muscular dystrophy (Figure 1, A and B). In the current study, we report that $\alpha \mathrm{LNNd}$ indeed repaired the skeletal muscle defects in $d y 2 \mathrm{~J}$ mice and enabled the assembly of nonpolymerizing laminins in a muscle $\mathrm{BM}$ in culture. These experiments provide a proof of concept for the development of a new treatment option for ambulatory-type MDC1A, while at the same time validating a mechanistic model of BM assembly with predictive value.

\section{Results}

Laminin and laminin-binding linker proteins. Previous work in vitro revealed that polymerization is mediated by the $\mathrm{N}$-terminal lami$\operatorname{nin} \alpha-, \beta$-, and $\gamma$-subunit LN domains that bind to each other to form a ternary node $(14,17-19,29)$. One study (15) described the biochemical and Schwann cell BM assembly characteristics of a recombinant $157-\mathrm{kDa}$ chimeric protein, designated $\alpha \mathrm{LNNd}$. This engineered chimeric protein consists of the mouse $\mathrm{N}$-terminal moiety of the laminin $\alpha 1$-short arm (LN and 4 adjacent LEa domains) fused to the nidogen-1 N-terminal domains G2 through G3 (Figure 1A). The G3 domain of $\alpha$ LNNd binds to the nidogen-1-binding locus (LEb3) on the $\gamma 1$-short arm of recombinant Lm111, adding an additional short arm. The $\alpha 1 \mathrm{LN}$ domain on this arm can substitute for the native $\alpha 2 \mathrm{LN}$ domain for polymerization when the $\alpha$-short arm is absent (Figure 1B). The chimeric protein also binds to collagen IV through the G2 and G3 domains of the nidogen moiety, with
G2 predicted to bind to perlecan (30), thereby preserving all known $\mathrm{BM}$ assembly functions that might otherwise be lost by replacing nidogen with $\alpha \mathrm{LNNd}$. To test the model of laminin polymerization in vivo, $\alpha \mathrm{LNNd}$-encoding cDNA was transgenically expressed in mice under the control of the muscle creatine kinase (MCK) promoter. $\alpha$ LNNd was detected as an approximately $157-\mathrm{kDa}$ protein in muscle (Figure 1C) and was incorporated into sarcolemmal BM as seen by bright immunostaining in muscle (Figure 1D).

Transgenic expression of $\alpha$ LNNd in dy2J mice. The $\alpha \mathrm{LNNd}$ transgene was bred into $d y^{2 J} /+$ mice (referred to herein as $\mathrm{Tg}+d y 2 J$ mice) for evaluation of the effect of $\alpha \mathrm{LNNd}$ in homozygous mutant mice. In $d y 2 J$ mice, a 57-residue-long part of an $\mathrm{N}$-terminal segment within the laminin $\alpha 2 \mathrm{LN}$ domain is deleted as a consequence of a splice donor defect $(27,28)$. This deletion was found to destabilize the $\alpha 2 \mathrm{LN}$ domain, such that it then readily degrades through proteolysis, making the laminin unable to polymerize (25). If the laminin assembly model is correct, expression of $\alpha \mathrm{LNNd}$ would be predicted to restore this polymerization deficit and ameliorate the muscular dystrophy.

Grip strength. Dystrophic mice showed signs of a hind limb gait abnormality by 3 weeks of age that was followed by weakness in all limbs. We evaluated this weakness by specific grip strength measurements (grams of peak force divided by mouse weight) as a function of age (Figure 2). At 3 weeks, we detected no significant reduction of grip strength in the forelimbs of dystrophic mice, whereas small differences were detected in the hind limbs and combined fore- and hind limbs. By 4 weeks, we found that forelimb grip strength was reduced in dystrophic mice to half that of control mice. In the presence of the transgene, only a small reduction in forelimb grip strength was detected compared with controls. By 6 weeks of age, dystrophic mouse grip strength had increased to approximately $60 \%$ of control levels, whereas $\mathrm{Tg}+d y 2 \mathrm{~J}$ mouse grip strength had increased to levels statistically indistinguishable from those of normal control mice. This set of relationships did not change through 11 weeks age. Although females weighed less than males in adulthood, no significant specific grip strength differences were found between the sexes (Supplemental Figure 1; supplemental material available online with this article; https:// doi.org/10.1172/JCI90854DS1). Also, no differences were noted among the 4 control genotypes. In addition to the quantitative differences, by 4 weeks of age, it was possible to distinguish between control and $d y 2 J$ mice by simply pulling mice away from a wire mesh gripped with the forelimbs: $d y 2 J$ mice were considerably weaker than were control mice, while control and $\mathrm{Tg}+d y 2 \mathrm{~J}$ mice had similar forelimb grip strength.

Hind limb grip strength measured in isolation was less than forelimb grip strength. As the mice aged between 3 and 11 weeks, $d y 2 J$ hind limb grip strength was reduced from 2.5 to $3.5 \mathrm{gm} / \mathrm{gm}$ to 1.5 to $2 \mathrm{gm} / \mathrm{gm}$. $\alpha \mathrm{LNNd}$ transgene expression partially improved grip strength through 7 weeks, but not thereafter. Loss of the transgene benefit corresponded to the development of permanent hind limb extension contractures, which are the result of prominent peripheral neuropathy, a phenotype that is not affected by expression of the transgene in skeletal muscle. When all limbs were evaluated together, the small initial reduction of grip strength in $d y 2 J$ mice became maximal by 5 weeks of age, with transgene expression associated with an improvement in grip strength of approximately $50 \%$ compared with that of controls, reflecting full recov- 
A

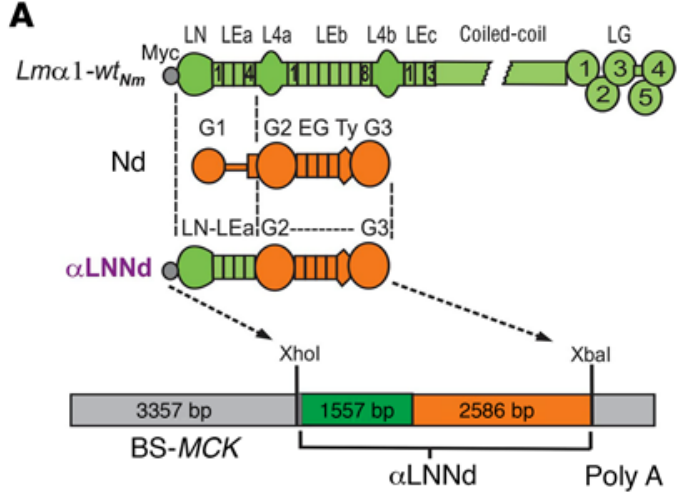

C

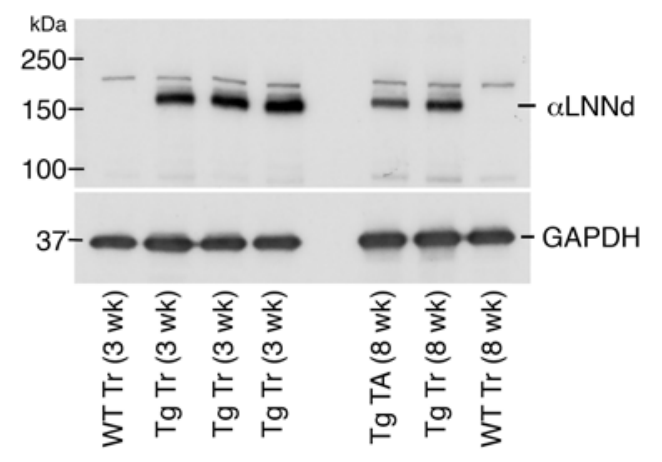

B

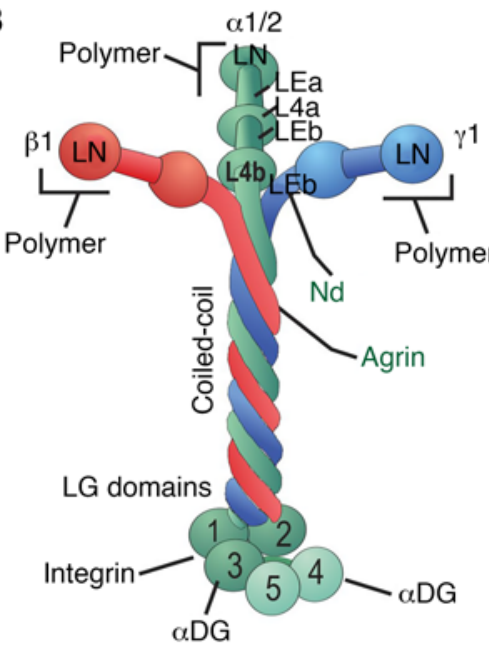

D

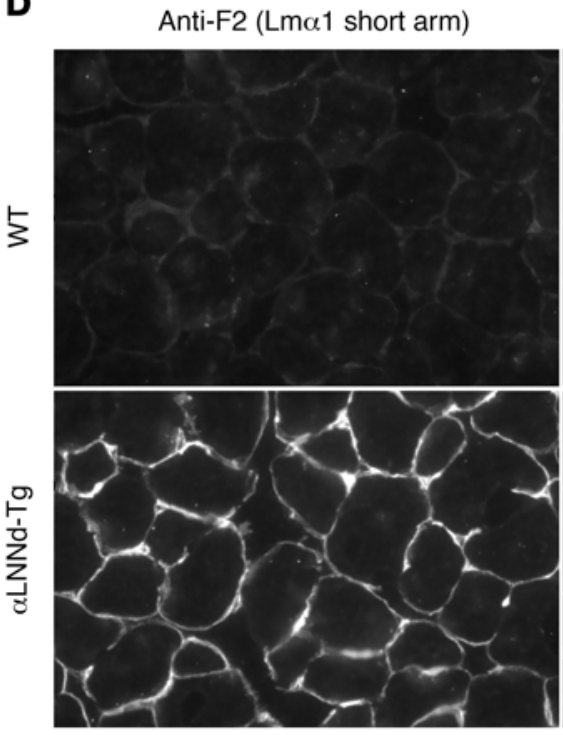

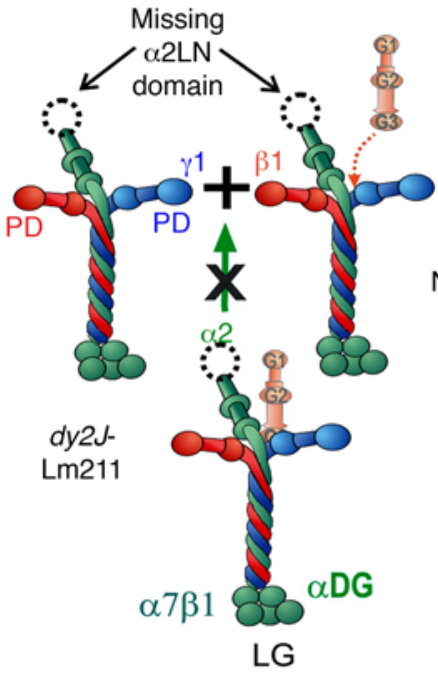

$$
\text { by }
$$

replaced by $\alpha$ LNNd

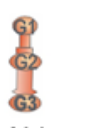

$\mathrm{Nd}$

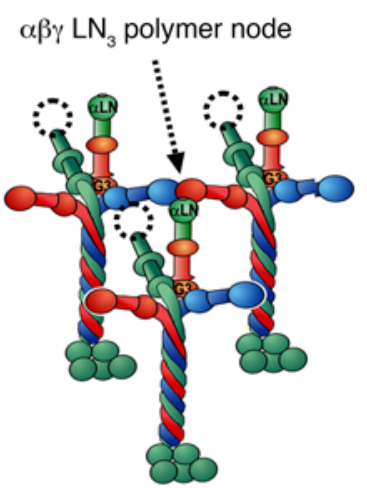

Figure 1. $\alpha$ LNNd transgene. (A) $\alpha$ LNNd is a chimeric protein consisting of murine $\mathrm{Lm} \alpha 1 \mathrm{LN}-\mathrm{LEa}$ domains fused to the C-terminal moiety of nidogen-1. Its corresponding cDNA was inserted into a vector driven by the MCK promoter and used to generate transgenic mice. (B) Muscle Lm211 and its homolog Lm111 bind to nidogen-1 (Nd), agrin, $\alpha \mathrm{DC}$, and $\alpha 7 \beta 1$ integrin. Polymerization results from the binding of the $L N$ domains (PD) of the $\operatorname{Lm} \alpha 2$ (or $\alpha 1$ ), $L m \beta 1$, and $L m \gamma 1$ subunits to form a ternary node at the $\mathrm{N}$-termini of each laminin. The $\alpha 2 \mathrm{LN}$ domain is defective in the dy2J mouse, greatly reducing self-assembly. $\alpha$ LNNd can compete with and replace native nidogen-1, binding to the Lm $\gamma 1$-LEb3 domain locus, to create a new short arm capable of binding to the other LN domains. (C) $\alpha$ LNNd protein expression was probed by immunoblotting of extracts from 3-and 8-week-old triceps (Tr) and tibialis anterior (TA) (goat anti-Lma1-LN Ab). Expression was detected in $\alpha$ LNNd-Tg but not WT muscle. (D) Immunostained sections of triceps muscle from 3-week-old WT and $\alpha \mathrm{LNNd-Tg} \mathrm{mice} \mathrm{revealed} \mathrm{a} \mathrm{sarcolemmal} \mathrm{BM} \mathrm{distribution}$ of $\alpha$ LNNd (anti-F2, reacting with Lma1LN-LEa) similar to that of Lma2 (rat anti-Lma2). Scale bar: $100 \mu \mathrm{m}$. ery of forelimb grip strength in the absence of substantial hind limb improvement. The full degree of muscle transgene-dependent restoration of forelimb function demonstrates the importance of laminin polymerization. It also indicates that the peripheral neuropathy contributes little to the $d y 2 J$ loss of forelimb strength. The limited improvement in hind limb strength, on the other hand and as discussed ahead, likely reflects the significant contribution of $d y 2 J$ sciatic nerve amyelination to hind limb function.
Muscle forelimb histology and morphometry. H\&E-stained $d y 2 J$ muscles examined in cross sections of the extensor carpi radialis longus and brevis revealed characteristic dystrophic changes including reduced overall cross-sectional size, small and variable myofiber size, increased presence of central nuclei, rounding of myofibers in cross sections, and weakly stained interstitial material separating myofibers (Figure 3). We detected histological abnormalities in $d y 2 J$ forelimb muscle at 3 to 4 weeks of age. By 11 weeks, we observed a 

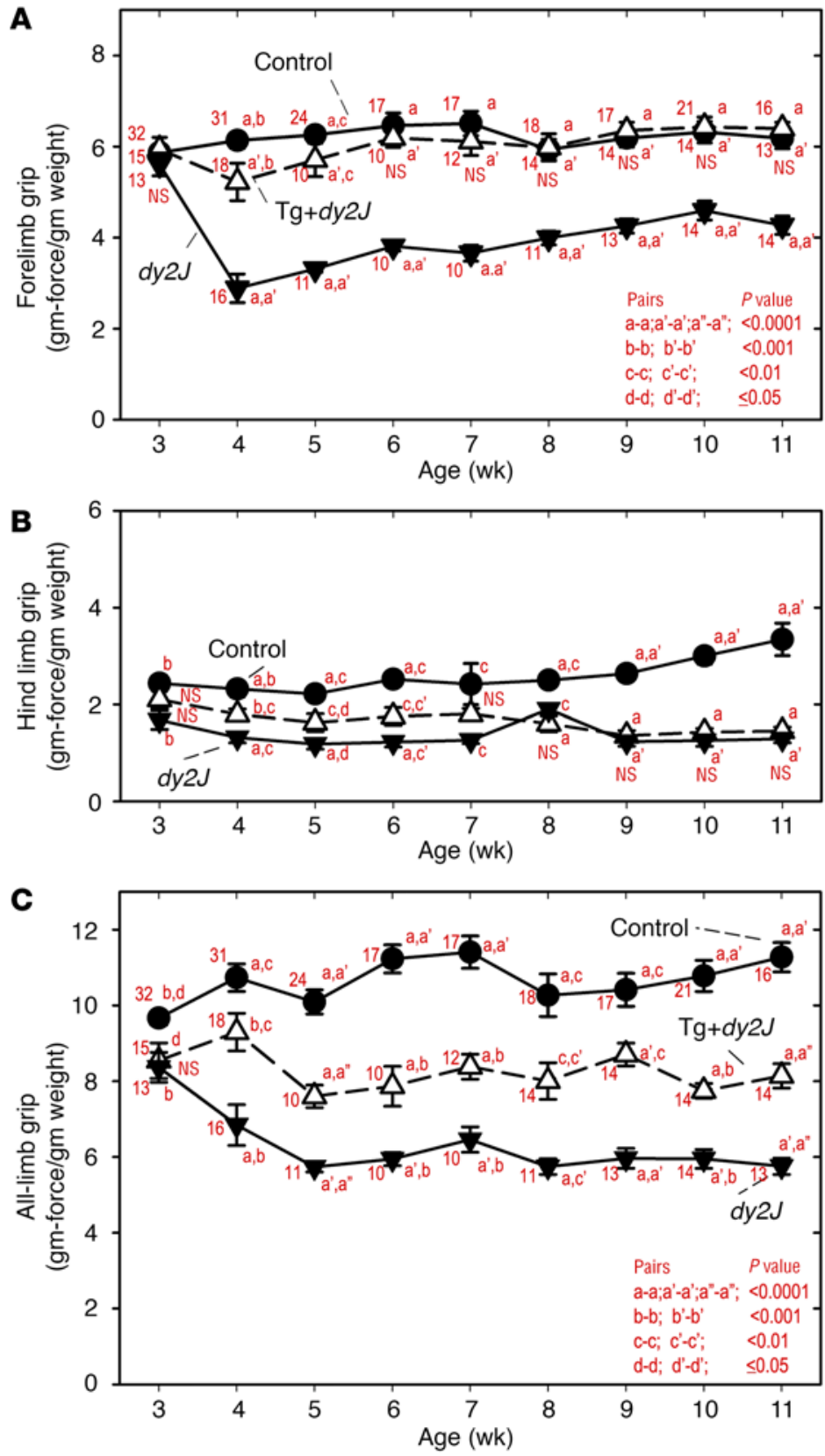

Figure 2. Mouse-specific grip strength. Forelimb (A), hind limb (B), and combined limb (C) measurements were conducted on control, dy2J, and $\alpha \mathrm{LNNd}$ plus Tg+dy2J mice between 3 and 11 weeks of age. Values shown represent the mean \pm SEM of individual averaged mouse sets of grip strength determinations divided by mouse mass. The number of mice used for each determination is indicated in red. Lowercase red letter pairs indicate the statistical significance ranges, which were determined by 1-way ANOVA with pairwise Holm-Sidak comparisons ( $P$ value ranges are indicated in the lower right corner of the top and bottom panels). NS, not significant. Dy2J mice showed reduced forelimb grip strength by 4 weeks of age. Grip strength was improved to near-control levels with the $\alpha \mathrm{LNNd}$ transgene and became indistinguishable from that of control mice by 6 weeks of age. For hind limbs, the transgene partially increased grip strength between 3 and 7 weeks, but not thereafter, corresponding to the emergence of hind limb contractures. Note that the peripheral nerve defect, which selectively affected the hind limbs, was not corrected, because a muscle-specific promoter was used to drive $\alpha$ LNNd expression. Combined (all-limb) grip strength was reduced in dy2J mice, with substantial improvement ( $50 \%)$ detected in the presence of the transgene. further increase in the extent of interstitial material that stained bright red with Picrosirius red, indicative of the increased collagen deposition seen in fibrosis. The presence of the transgene greatly improved the H\&E- and Picrosirius red-stained morphology, such that it closely resembled that of control muscle, with the exception of some residual central nuclei. Using morphometry, we quantified the dystrophic changes and compared them with those in mice expressing the transgene. Of the parameters measured, all but the number of centralized myonuclei were normalized to control levels by expression of the transgene. These included the frequency of distribution of myofiber areas (Figure 3G), average total cross-sectional area (Figure 3G inset), overall myofiber size (Figure 3H), myofiber count (Figure 3I), and degree of fibrosis (Picrosirius red fluorescence; Figure 3K). The average fraction of myofibers with central nuclei was reduced from approximately 0.2 to 0.06 compared with
0.01 for control muscles (Figure 3J). Other forelimb muscles showed similar pathology and improvement in the presence of the transgene.

We also examined the hind limb quadriceps muscle rectus femoris (Supplemental Figure 2). The same general improvements were observed, with several exceptions. First, the total number of myofibers was similar among control, $d y 2 J$, and $\mathrm{Tg}+d y 2 J$ muscles (Figure $3 \mathrm{H})$.Second, the reduction in the fraction of central nuclei was substantial but not as great $(\sim 0.22$ in $d y 2 J$ muscle and 0.10 in $\mathrm{Tg}+d y 2 J$ muscle), and the distribution of myofiber areas was greatly improved, but not to the same degree as that observed in control muscle. Thus, hind limb muscles were improved by the expression of $\alpha \mathrm{LNNd}$, as were forelimb muscles, although these improvements did not reach control levels. Some of the differences observed in the forelimb muscle may be accounted for by the confounding effects of the hind limb paralysis. 

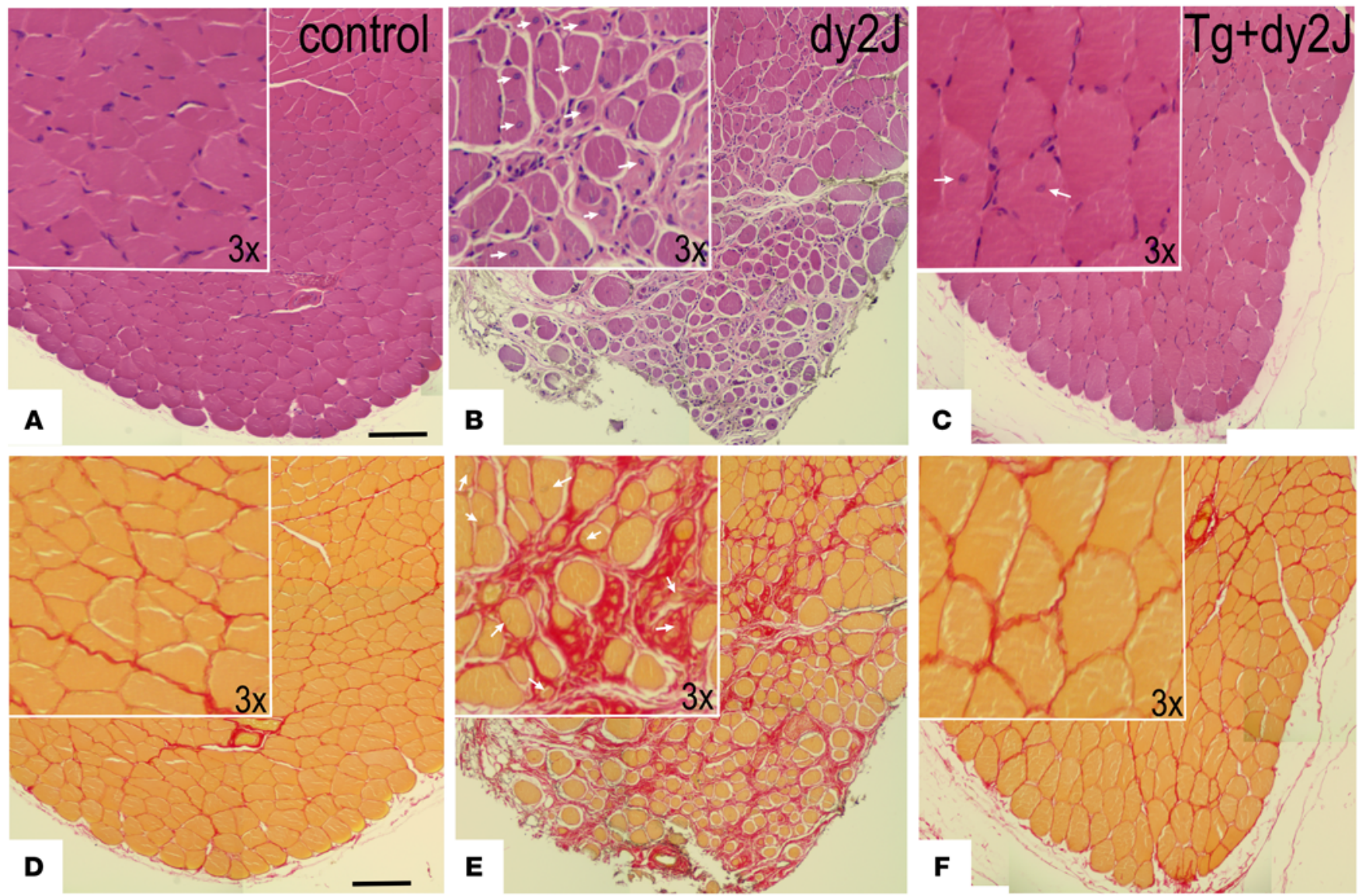

G

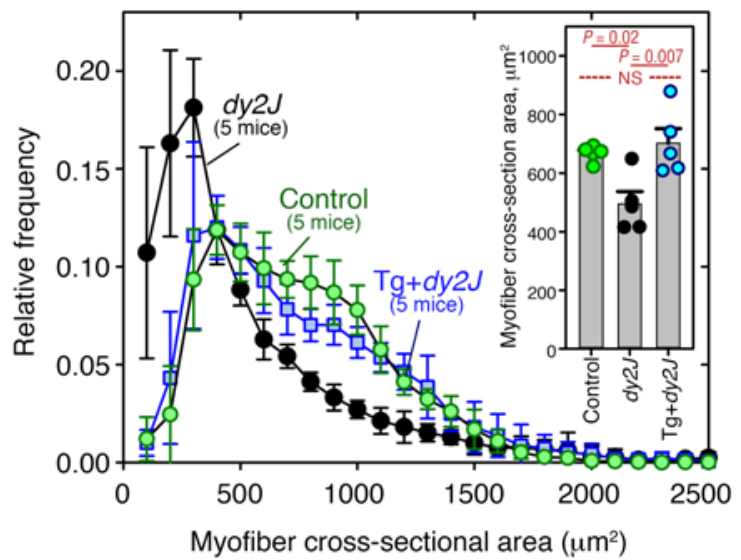

$\mathbf{H}$

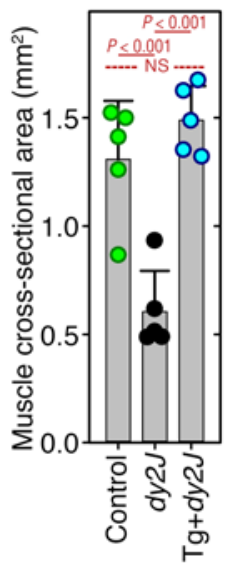

I

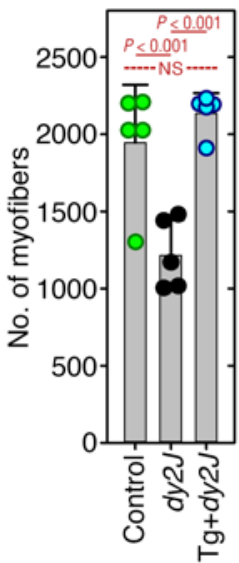

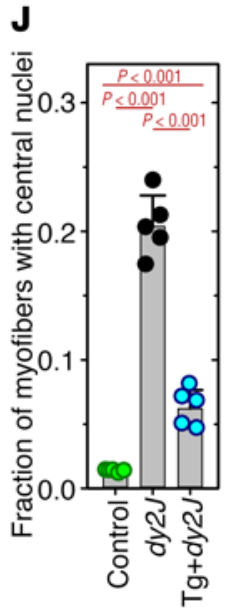

$\mathbf{K}$

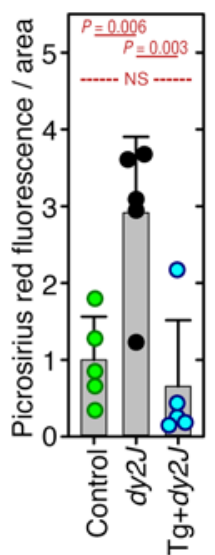

Figure 3. Forelimb muscle histology. (A-C) H\&E staining of cross sections of the corresponding region of 11-week-old extensor carpi radialis muscle. (D-F) Picrosirius red collagen staining of adjacent sections. White arrows indicate examples of central nuclei in magnified insets (original magnification, $\times 3$ ). The morphology of rounded, variable-sized myofibers with increased interfiber collagen-positive staining, characteristic of dystrophic muscle, was improved by the transgene to more closely resemble control muscle morphology. Scale bars: $100 \mu \mathrm{m}$. (G) Histogram showing the distribution of cross-sectional myofiber areas comparing control, dy2J, and Tg+dy2J mice ( $n=5$ mice/condition; data represent the average \pm SD). Inset graph in $\mathbf{G}$ shows the overall myofiber cross-sectional area (average \pm SEM, $n=5$ /condition). Histogram plot shows a smaller size distribution for dy2J muscle but a normal distribution and overall average for Tg+dy2J muscle. (H) Average (mean $\pm \mathrm{SD}, n=5$ mice/condition) of the sum of myofiber cross-sectional areas. The area reduction in dy2J muscle was corrected by the transgene. (I) Number of myofibers per muscle (average $\pm \mathrm{SD}, n=5 /$ condition). The number of myofibers was reduced in dy2 $\mathrm{J}$ muscle and increased to levels indistinguishable from those of control muscle with the transgene. (J) The fraction of myofibers (average \pm SD, $n=5 /$ condition) with central nuclei (regeneration marker), elevated in $d y 2$ J muscle, was decreased in the presence of the transgene. (K) Picrosirius red fluorescence, a collagen stain, was reduced to levels indistinguishable from those in control muscle (average $\pm S D, n=5$ /condition). Individual mouse values are superimposed on the average bars in panels G-K. $P$ value determined in G-K by 1-way ANOVA followed by pairwise Holm-Sidak test. 

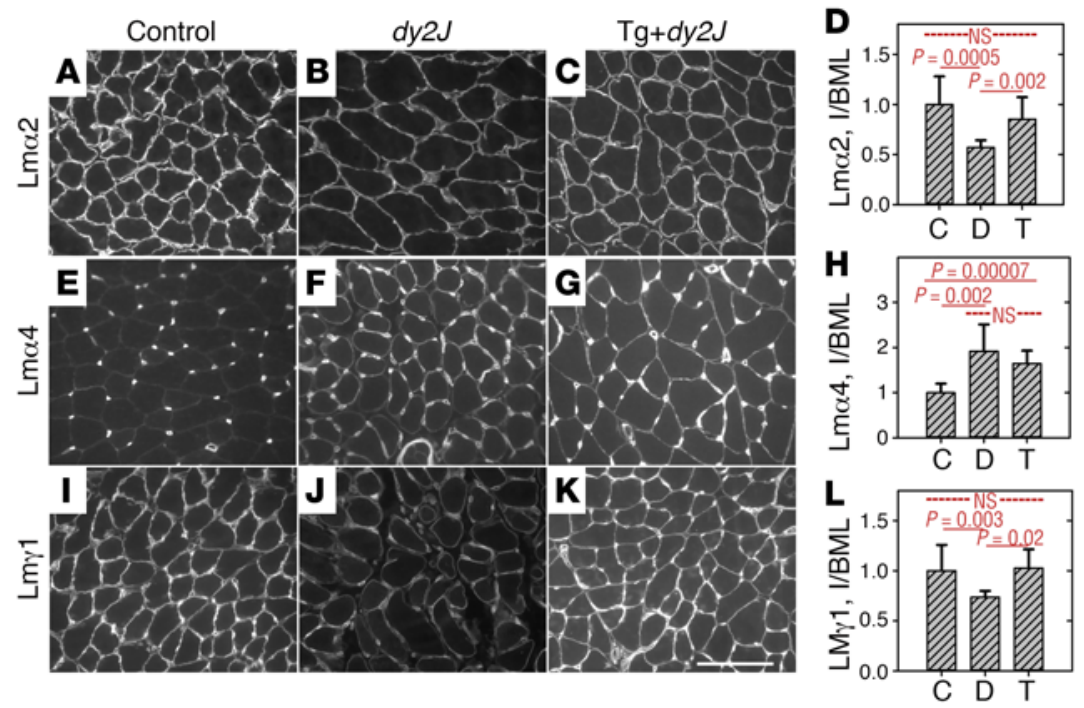

Figure 4. Muscle laminins. (A-L) Immunofluorescence microscopy. Frozen sections of 4-week-old forelimb triceps brachii from control, dy2J, and Tg+dy2J littermates were rinsed, fixed, and immunostained with an $A b$ specific for the $L m \alpha 2$ (L4b domain, A-D), Lm $\alpha 4$ (E-H), and Lm $\gamma 1$ (I-L). Original magnification, $\times 20$ objective; scale bar: $100 \mu \mathrm{m}$. $(\mathbf{D}, \mathbf{H}$, and $\mathbf{L}$ ) Graphs of the overall degree of immunofluorescence difference as estimated by segmentation (sum of pixel intensities minus background) and divided by the traced sum of BM lengths (I/BML) for the above Abs. Data represent the average \pm SD for 8 to 12 fields per condition. $P$ values were determined by 1-way ANOVA with Holm-Sidak pairwise comparisons. C, control; D, dy2); T, $\mathrm{Tg}+d y 2 J$. Reductions in $\mathrm{Lm} \alpha 2$ and $\mathrm{Lm} \gamma 1$ subunit levels were seen in homozygous dy2J muscle and were increased in $\mathrm{Tg}+d y 2 J$ muscle. $\operatorname{Lm} \alpha 4$ levels were increased in both $d y 2 J$ and $\mathrm{Tg}+d y 2 J$ muscle.

Laminin in muscle. Sections of 4-week-old triceps from littermate mice were examined by immunostaining to detect Lm $\alpha 2$, Lm $\alpha 4$, and Lm $\gamma 1$ subunits at a stage when fibrosis was still minimal (Figure 4). The images and graphs in Figure 4 show decreased levels of $\operatorname{Lm} \alpha 2$ and $\operatorname{Lm} \gamma 1$ in $d y 2 J$ muscle relative to the levels detected in control muscle, which is in agreement with previous observations (31). The $\alpha \mathrm{LNNd}$ transgene increased laminin subunits to levels that were not significantly different from those of controls (Figure 4, D, H, and L). Lm411 levels, on the other hand, were increased in both $d y 2 J$ and $\mathrm{Tg}+d y 2 J$ muscles relative to the levels detected in controls, and this increase was noted in capillaries and, to a lesser degree, in adjacent sarcolemma. Lm $\alpha 5$ (Supplemental Figure 3) was similarly localized in the microvasculature for all conditions. Sarcolemmal collagen IV was similar for all conditions, as was $\alpha \mathrm{DG}$, while integrin $\beta 1$ was increased in focal areas of $d y 2 J$ muscle. We found that $\operatorname{Lm} \alpha 4 / \operatorname{Lm} \alpha 2$ ratios were elevated in peripheral nerves in $d y 2 J$ and $\mathrm{Tg}+d y 2 J$ mice. We also examined muscle ultrastructure. In 4-week-old triceps, the sarcolemma appeared similar among control, $d y 2 J$, and $\mathrm{Tg}+d y 2 \mathrm{~J}$ mice (data not shown). By 8 weeks, we observed BM attenuations and interruptions and increases in adjacent interstitial collagen fibrils in $d y 2 J$ muscle, whereas $\mathrm{Tg}+d y 2 J$ sarcolemmal BMs and stroma were similar to those of controls. The transgene therefore appeared protective of $d y 2 J$ muscle ultrastructure.

Laminins were sequentially extracted from 4-week-old forelimb skeletal muscle and analyzed as combined total laminins, buffer (soluble intracellular and extracellular laminins), and BM (matrix) laminins (consisting of collagenase plus EDTA plus SDS fractions as described in the Methods section) in sandwich ELISA assays to detect heterotrimeric laminins and by quantitative reverse transcriptase PCR (qRT-PCR) to compare mRNA levels (Figure 5). The sandwich assays (Figure 5, A-K) showed that total $\mathrm{Lm} 211$ was reduced and total $\mathrm{Lm} 411$ was increased in $d y 2 J$ muscle compared with control muscle, with no significant change between $d y 2 J$ and $\mathrm{Tg}+d y 2 J$ muscle (Figure $5, \mathrm{~A}-\mathrm{D}$ ). In contrast, we found that $\mathrm{Lm} 211, \mathrm{Lm} 411$, and $\mathrm{Lm} \beta 1 \gamma 1$ in the matrix fractions were increased in $\mathrm{Tg}+d y 2 J$ muscle relative to $d y 2 J$ muscle (Figure $5, \mathrm{E}-\mathrm{J}$ ). These experiments suggest that the transgene does not affect Lm211 and
Lm411 total levels but increases their relative contribution to the matrix. We then analyzed RNA extracts from forelimb muscle from control, $d y 2 \mathrm{~J}$, and Tg+dy2J mice by qRT-PCR (Figure 5, L-R). Transcripts encoding Lma2 and Lm $\gamma 1$ (Lama2 and Lamc1, respectively) remained constant for all conditions (Figure 5, L and N). In contrast, we found that transcript levels of the gene encoding Lma4 (Lama4) were doubled in $d y 2 J$ muscle compared with levels in control muscle and remained elevated in the presence of the transgene (Figure $5 \mathrm{M}$ ). Transcript encoding integrin $\alpha 7$ or $\beta 1$ increased in $d y 2 \mathrm{~J}$ muscle and returned to control levels in the presence of the transgene, whereas dystroglycan mRNA levels remained constant. Fibronectin, an inflammatory marker, was increased in $d y 2 J$ muscle compared with control and transgene muscle.

We also asked what fraction of muscle laminins is bound to $\alpha \mathrm{LNNd}$ instead of to endogenous nidogen, the normal laminin partnering protein. Combined forelimb muscle extracts from 4-weekold mice were probed by immunoprecipitation and immunoblotting to examine the relationship of $\alpha \mathrm{LNNd}$ to nidogen-1, and the laminin bound to each (Figure 6). The ratio of $\alpha \mathrm{LNNd}$ to nidogen-1 was estimated to be $1: 2$, with approximately half of the $\beta 1 \gamma 1$-laminins bound to $\alpha \mathrm{LNNd}$ and the remainder bound to nidogen-1.

Laminin and collagen IV accumulation on C2C12 myotubes, an in vitro model of $B M$ assembly. We asked whether the changes in laminin observed in the different muscle BMs could be explained by the intrinsic assembly properties of polymerizing, nonpolymerizing, and polymerization-rescued laminins. To address this question, recombinant laminins were allowed to assemble on the lawns of fused mouse C2C12 myotubes. While applying BM components to myotubes provides imperfect modeling of myomatrix assembly in vivo, the approach has proven useful for examining how initially soluble BM components can assemble on cell surfaces that possess relevant $\alpha 7 \beta 1$ integrin and $\alpha D G$ receptors, with negligible endogenous expression of BM components (32). The culture system includes the coassembly of nidogen and collagen IV, like the system used to study BM assembly on Schwann cells (14). Lm111 lacking either the entire $\alpha$-short arm or only the $\mathrm{LN}$ domain was chosen for the analysis, because (a) Lm111 is closely related to neuromuscular Lm211, and both proteins polymerize and bind to nidogen, $\alpha 7 \beta 1$ 
integrin, and $\alpha \mathrm{DG}$; (b) Lm111 can fully rescue the severe dystrophy and peripheral neuropathy of the Lama2-KO mouse $(33,34)$; and (c) a collection of $\alpha 1$-laminins bearing deletions and point mutations that affect specific functions have been generated, characterized, and previously used to dissect laminin functions in the laboratory $(14,35,36)$. In addition, the assembly of WT recombinant Lm211, Lm411, and Lm511 was compared with that of Lm111.

Lm111 and nidogen-1 were evaluated at a concentration of 28 $\mathrm{nM}$, a condition found to approach saturation of accumulation, while collagen IV was evaluated at $14 \mathrm{nM}$, the highest soluble, usable concentration $(19,37)$. We used BM component cell-surface accumulation to estimate assembly $(14,15,32)$ and observed that Lm111 accumulated on myotubes in the absence of other added components, while nidogen and collagen IV did not assemble in the absence of laminin (Supplemental Figure 4, A-E). Furthermore, nidogen binding to laminin $(14,38)$ was required for collagen IV accumulation. These findings were qualitatively similar to observations made on cultured Schwann cells (14). The fine pattern of surface laminin in the myotubes was similar to that observed with EHS-Lm111 and thought to represent a precostameric distribution (32). Laminin accumulation approached a maximum value at approximately $30 \mathrm{nM}$, whereas collagen IV achieved a maximum value at approximately $3 \mathrm{nM} \mathrm{Lm111} \mathrm{(Supplemental} \mathrm{Figure} \mathrm{4E).}$

Lm211 is the principal laminin of the muscle's sarcolemmal $\mathrm{BM}$. When the $\alpha 2$-subunit is absent, the $\alpha 4$-subunit, normally present in the muscle capillaries, appears in the sarcolemma $(22,39)$. In order understand muscle isoform assembly, we evaluated Lm211, Lm411, and Lm511 (the latter being a microvascular laminin with limited sarcolemmal expression) accumulation on myotubes (Supplemental Figure 4, F-M). We found that Lm211 and Lm511, both polymerizing laminins with LG domain-mediated adhesion (16), accumulated on myotubes at similar levels. However, we detected almost no myotube Lm411 accumulation, even when it was coincubated with an equimolar concentration of $\alpha \mathrm{LNNd}$ (Supplemental Figure 4, G and $\mathrm{H}$ ), a finding that may be explained by weak receptor binding of Lm411 $(12,23)$. Comparable collagen levels were detected for the polymerizing laminins Lm111 and Lm211, whereas Lm411 and Lm411 plus $\alpha$ LNNd did not show accumulation.

We then examined the effect of $\alpha \mathrm{LNNd}$ on nonpolymerizing recombinant Lm111 and collagen IV (Figure 7). Lma $\Delta \mathrm{LN}-\mathrm{L} 4 \mathrm{~b}$ lacks an $\alpha 1$-short arm (with its critical $\alpha 1 \mathrm{LNd}$ domain) but possesses LG domains that bind well to $\alpha \mathrm{DG}$ and integrin $\alpha 7 \beta 1$ (14). Lm $\alpha \Delta \mathrm{LN}-\mathrm{L} 4 \mathrm{~b}$ accumulated on myotubes at a 2- to 3-fold reduced level compared with WT Lm111. When Lm $\alpha \Delta \mathrm{LN}-\mathrm{L} 4 \mathrm{~b}$ was added to myotubes with an equimolar concentration of $\alpha \mathrm{LNNd}$, we detected accumulation at levels equal to those of WT Lm111. In contrast, we observed no alteration of WT Lm111 when it was coincubated with $\alpha \mathrm{LNNd}$, indicating that duplication of the $\alpha \mathrm{LN}$ domain had no significant effect on laminin assembly. We found that accumulation of collagen IV was also impaired, but less so, in the Lm $\alpha \Delta \mathrm{LN}-\mathrm{L} 4 \mathrm{~b}$ mutant. A mixture of $\alpha \mathrm{LNNd}$ and Lm $\alpha \Delta \mathrm{LN}-\mathrm{L} 4 \mathrm{~b}$ restored the accumulation of collagen IV to levels slightly below those observed with WT Lm111 (Figure 7, D and E). These experiments show that $\alpha \mathrm{LNNd}$ is capable of compensating for the loss of the LN domains in Lm111 to mediate binding to myotubes.

We evaluated the concentration dependency of laminin assembly for WT Lm111 and nonpolymerizing Lm $\alpha \Delta \mathrm{Ln}-\mathrm{L} 4 \mathrm{~b}$ and
Lm $\alpha \Delta \mathrm{LN}$ (Supplemental Figure 5, A-F). Lm $\alpha \Delta \mathrm{LN}$, a second modified laminin that we examined, is similar to $d y 2 J \mathrm{Lm} 211$, in that both selectively lack an $\alpha \mathrm{LN}$ domain and bind well to $\alpha \mathrm{DG}$ and integrin $\alpha 7 \beta 1$. WT Lm111 accumulation approached a plateau at concentrations above $20 \mathrm{nM}$ (Supplemental Figure 5, A and B). We found that Lm $\alpha \Delta \mathrm{LN}-\mathrm{L} 4 \mathrm{~b}$ accumulation was below that of WT Lm111 at all concentrations examined. $\alpha \mathrm{LNNd}$ increased $\mathrm{Lm} \alpha \Delta \mathrm{LN}$ L4b accumulation to levels nearly identical to those of WT Lm111 (Supplemental Figure 5, C and D). The nonpolymerizing laminin (Lm $\alpha \Delta \mathrm{LN}$ ) that selectively lacks the LN domain also accumulated poorly compared with WT laminin (Supplemental Figure 5, E and F). $\alpha$ LNNd substantially increased this accumulation as well.

$\alpha L N N d$-nidogen competition. The degree of $\alpha \mathrm{LNNd}$ occupancy on laminins (Figure 6) may in part result from competition between transgenic $\alpha \mathrm{LNNd}$ and endogenous nidogen- 1 for the same binding locus in the laminin $\gamma 1$-short arm, a possibility, given a report that the nidogen/laminin molar ratio in muscle is 2:1 (40). We examined this in C2C12 myotubes (Supplemental Figure $5, \mathrm{G}$ and $\mathrm{H}$ ) and found that when $\alpha \mathrm{LNNd}$ was maintained constant and the nidogen- 1 concentration increased over that of $\alpha \mathrm{LNNd}$, laminin accumulation progressively declined. The limited extent of this reduction may be a consequence of a favored assembly for those laminins that polymerize.

\section{Discussion}

$\alpha \mathrm{LNNd}$ is a laminin-binding protein that can restore polymerization activity in laminins with defective $\alpha$-subunit short arm domains. Its C-terminal globular domain binds to the 1 subunit of laminins at the nidogen-binding locus in domain LEb3, which is located near the point of the short arm trifurcation to provide a synthetic short arm with an active $\alpha 1 \mathrm{LN}$ domain at its N-terminal tip. The engineered protein also contains binding sites for collagen IV and perlecan, retaining the known BM activities of nidogen.

In this study, we evaluated whether $\alpha \mathrm{LNNd}$ can restore muscle function and structure in dystrophic $d y 2 J$ mice by driving expression of a transgene coding for the fusion protein using a muscle-specific promoter. This analysis revealed that $\alpha \mathrm{LNNd}$ expression was strongly beneficial to the dystrophic mice, repairing grip strength and muscle histology. The improvement was especially evident in forelimb muscles that were largely unaffected by the accompanying and uncorrected Lm $\alpha 2$-deficient peripheral nerve defect. $\mathrm{Tg}+d y 2 \mathrm{~J}$ forelimb grip strength, reduced in $d y 2 J$ mice, was indistinguishable from that of control mice by 6 weeks of age. Complementing forelimb grip strength improvements, $\mathrm{Tg}+d y 2 \mathrm{~J}$ forelimb muscle was essentially indistinguishable from that of control forelimb muscle in terms of myofiber architecture, size, shape, and numbers, as well as network-like endomysial collagen distribution. The only difference we discerned was that the fraction of myofibers with central nuclei was not completely restored to control levels. We found that the phenotypic benefit of the transgene in $d y 2 \mathrm{~J}$ muscle was accompanied by an increase in total $\gamma 1$-laminins and Lm211 in sarcolemmal BM as detected by immunostaining and biochemical means. We also noted an increase in Lm411, a minor laminin that was increased by 2-fold in $d y 2 J$ muscle. The increase, noted at even higher levels in a severe laminin-deficient dystrophy (22), was thought to be driven by increased transcription, measured in $d y 2 \mathrm{~J}$ muscle as a 2 -fold 

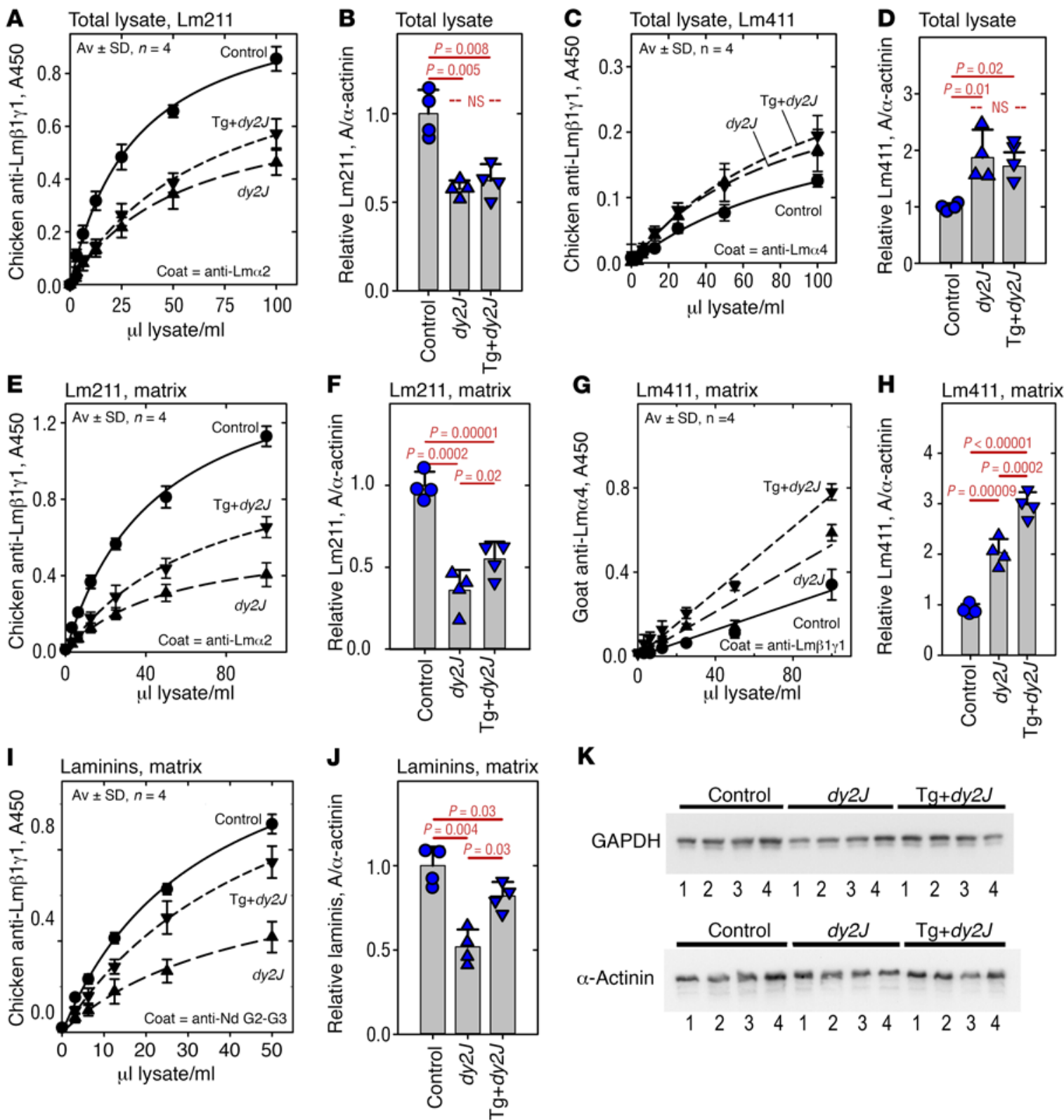

$\mathbf{K}$
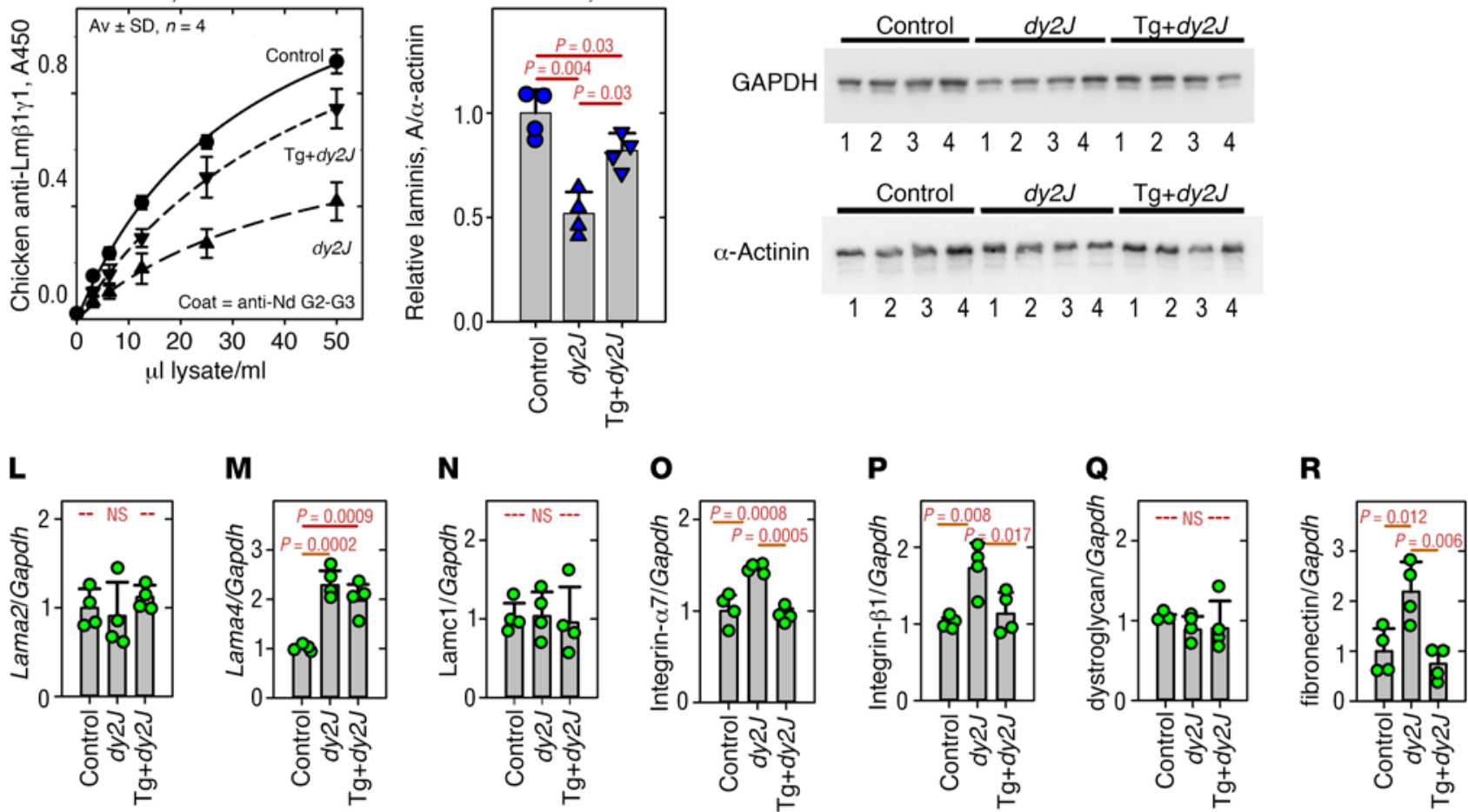
Figure 5. Muscle extracts. (A-K) Sandwich ELISA determination of laminin content in forelimb muscle. ELISA plates coated with rabbit polyclonal anti-Lma2

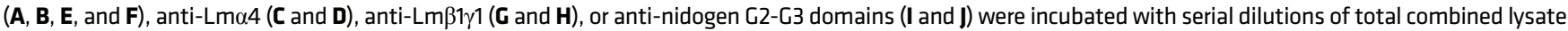
fractions (A-D) or "matrix" (collagenase plus EDTA plus SDS) fractions (E-J) extracted from muscle from four 4-week-old control (+/+ and dy2J/+), dy2J-mutant,

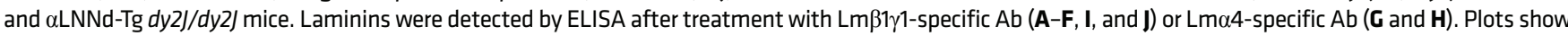
the relative average laminin absorbance ( \pm SD, $n=4$ /condition) for Lm211 (total, B), Lm411 (total, D), Lm211 (matrix, F), Lm411 (matrix, H), and total nidogen-bound laminins (matrix, J). (K) CAPDH and $\alpha$-actinin blots for each mouse muscle shown, with the latter used as a myofiber denominator. Lm211 was reduced, while Lm411 was increased in dy2J muscle lysates compared with control muscle total lysates, with no significant change in transgenic mouse muscle lysates. However, the pooled matrix fractions, after removal of soluble and loosely bound protein, contained significant increases of Lm211 and nidogen-binding laminins in Tg+dy2J muscle compared with dy2J muscle. To relate laminin levels to muscle $\alpha$-actinin, the averaged A450/lysate volume ratios in A, C, E, G, and I were divided by the relative $\alpha$-actinin immunoblot (K) intensities (A/ $\alpha$-act), shown in the corresponding plots in B, D, F, H, and J. (L-R) Changes in relative muscle mRNA by qRT-PCR $(\Delta \Delta \mathrm{Ct})$ at 4 weeks revealed that Lama2, Lamc1, and dystroglycan levels were not significantly different in WT, dy2), or Tg+dy2J muscle. Lma4 (Lama4) levels were similarly increased in $d y 2 J$ and $T g+d y 2 J$ muscle (but not increased in Tg+dy2J muscle relative to $d y 2 J$ muscle alone), while integrins $\alpha 7$ and $\beta 1$ and fibronectin (inflammatory marker) were increased in dy2J muscle and reduced in Tg+dy2J muscle. The presence of $\alpha$ LNNd did not change the Lama4 mRNA levels in WT mice. Average and SD plots are shown ( $n=4$ mice/condition in L-P and $\mathbf{R} ; n=3$ for control and $n=4$ for dy2J and Tg+dy2) mice in $\mathbf{Q}$ ). Graphs also show individual mouse values (colored circles) superimposed on bars. Statistical significance was determined by 1-way ANOVA with Holm-Sidak comparisons. Av, average.

increase in the subunit mRNA. A further increase in Tg+dy2J muscle appeared largely confined to capillary BMs and may not have materially contributed to the improved $d y 2 J$ muscle phenotype.

The Lma2 subunit is expressed in both muscle endomysium and peripheral nerve Schwann cell endoneurium. A reduction of the Lm $\alpha 2$ subunit in Schwann cells, as seen in $d y 2 J$ mice, causes a failure of radial sorting and amyelination, with forelimb nerves being less affected than hind limb nerves (41). The hind limb gait defect resulting from a selective Schwann cell ablation of the LAMC1 gene (PO promoter) is similar to that seen in $d y 2 J$ mice and consists of prominent hind limb weakness, followed by paralysis and permanent contractures, with little impairment of the forelimbs $(42,43)$. Minimal involvement of amyelination in forelimb function can further be deduced from the complete transgene-driven recovery of normal forelimb grip strength. Since the MCK promoter only enabled $\alpha \mathrm{LNNd}$ expression in muscle, dystrophic Lm211 was not altered in $\mathrm{Tg}+d y 2 J$ endoneurium (Supplemental Figure 3C), and hind limb grip strength was only partially improved in the time frame prior to the onset of contractures. It follows that the partial persistence of small myofibers in the $\mathrm{Tg}+d y 2 J$ hind limb histology may simply reflect neuropathic disuse atrophy. However, it is unclear whether persistent amyelination can also lead to a small degree of muscle fibrosis, as noted in the hind limb, or, instead, whether hind limb muscle requires a higher level of laminin repair than does forelimb muscle.

We evaluated the assembly of WT and nonpolymerizing laminins on cultured myotubes in tissue culture and compared this with the changes observed in dystrophic and transgenic/dystrophic mice. This in vitro analysis confirmed that BM assembly requires laminin assembly on cell surfaces and that the collagen accumulation substantially depends on a nidogen bridge between laminin and collagen. Only a relatively small concentration of laminin was required to obtain maximal collagen IV accumulation. This relationship may explain why, in contrast to laminin, major changes in collagen IV immunostaining were not noted in control, $d y 2 J$, and $\mathrm{Tg}+d y 2 J$ muscle. Strikingly, we found that efficient laminin cell-surface assembly was dependent on laminin polymerization. In the absence of polymerization activity, laminin accumulation was reduced by as much as 3 -fold over a wide concentration range. When $\alpha$ LNNd was added in equimolar amounts to nonpolymerizing laminin, laminin accumulation increased to levels the same or nearly the same as WT polymerizing laminin. We also found that the laminin isoforms Lm211 and Lm511, possessing polymerization- competent short arms and receptor-interactive LG domains, assembled to similar degrees on myotubes. In contrast, the compensatory laminin isoform Lm411, which lacks an $\alpha$-short arm and has poor LG-binding interactions, did not assemble on myotubes in the presence of $\alpha \mathrm{LNNd}$. This provided evidence that both conditions of LN-mediated polymerization and LG-mediated anchorage are required for efficient laminin assembly.
A

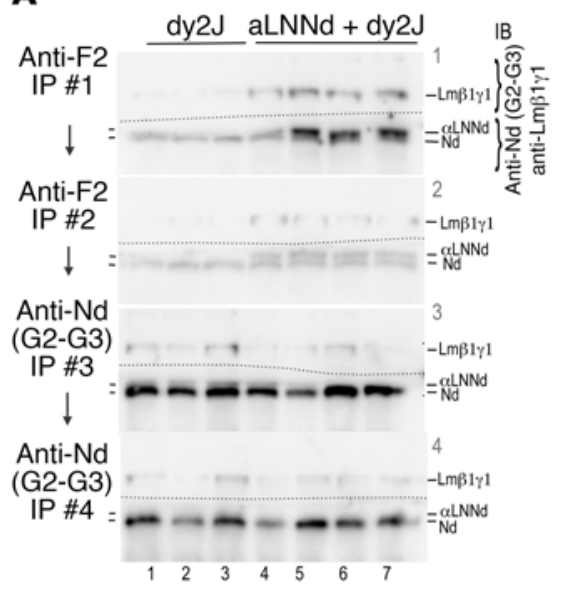

B

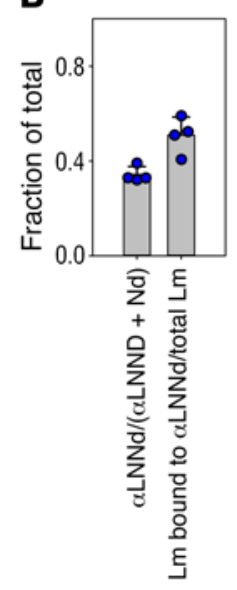

Figure 6. $\alpha$ LNNd and nidogen-1 in muscle. (A) Combined extracts (B plus [ plus E) from 4-week-old forelimb dy2] and $\mathrm{Tg}+d y 2$ ] muscle were immunoprecipitated twice with $\alpha$ LNNd-specific Ab (anti-F2, IP \#1 and IP \#2). The $\alpha$ LNNd-depleted supernatants were then immunoprecipitated with anti-nidogen G2-G3 (IP \#3 and IP \#4). Precipitates, following SDS-PAGE and membrane transfer, were immunoblotted with anti-Lm $\beta 1 \gamma 1$ (upper cut membrane, dotted cut line shown) and anti-nidogen G2-G3 (lower cut membrane). $\alpha$ LNNd was detected only in the Tg+dy2J muscle. (While a small amount of nidogen was present in the anti-F2 immunoprecipitates of the dy2] control muscle, this band was similarly seen in immunoblots precipitated with anti-lgY agarose beads alone and deduced to result from nonspecific nidogen binding to beads). Following $\alpha$ LNNd depletion, isolated nidogen was immunoprecipitated with anti-nidogen C2-G3 Ab. The upper $\alpha$ LNNd band was distinguished from the adjacent nidogen band to determine the relative amounts in the lysates (IP \#1 plus IP \#2). To estimate the fraction of $\beta 1 \gamma 1$-laminins bound to $\alpha$ LNNd and to nidogen-1, the laminin band intensities were multiplied by the fraction of $\alpha$ LNNd and nidogen- 1 in each blot to determine the distribution and then summed. (B) Plot (average $\pm \mathrm{SD}$, $n=4$ mice/condition, $P=0.007$, by 2 -tailed $t$ test) of the calculated fraction of $\alpha$ LNNd relative to the total of $\alpha$ LNNd plus nidogen and the fraction of $\beta 1 \gamma 1$ laminins bound to $\alpha$ LNNd relative to total $\beta 1 \gamma 1$ laminins. Lm, laminins. 

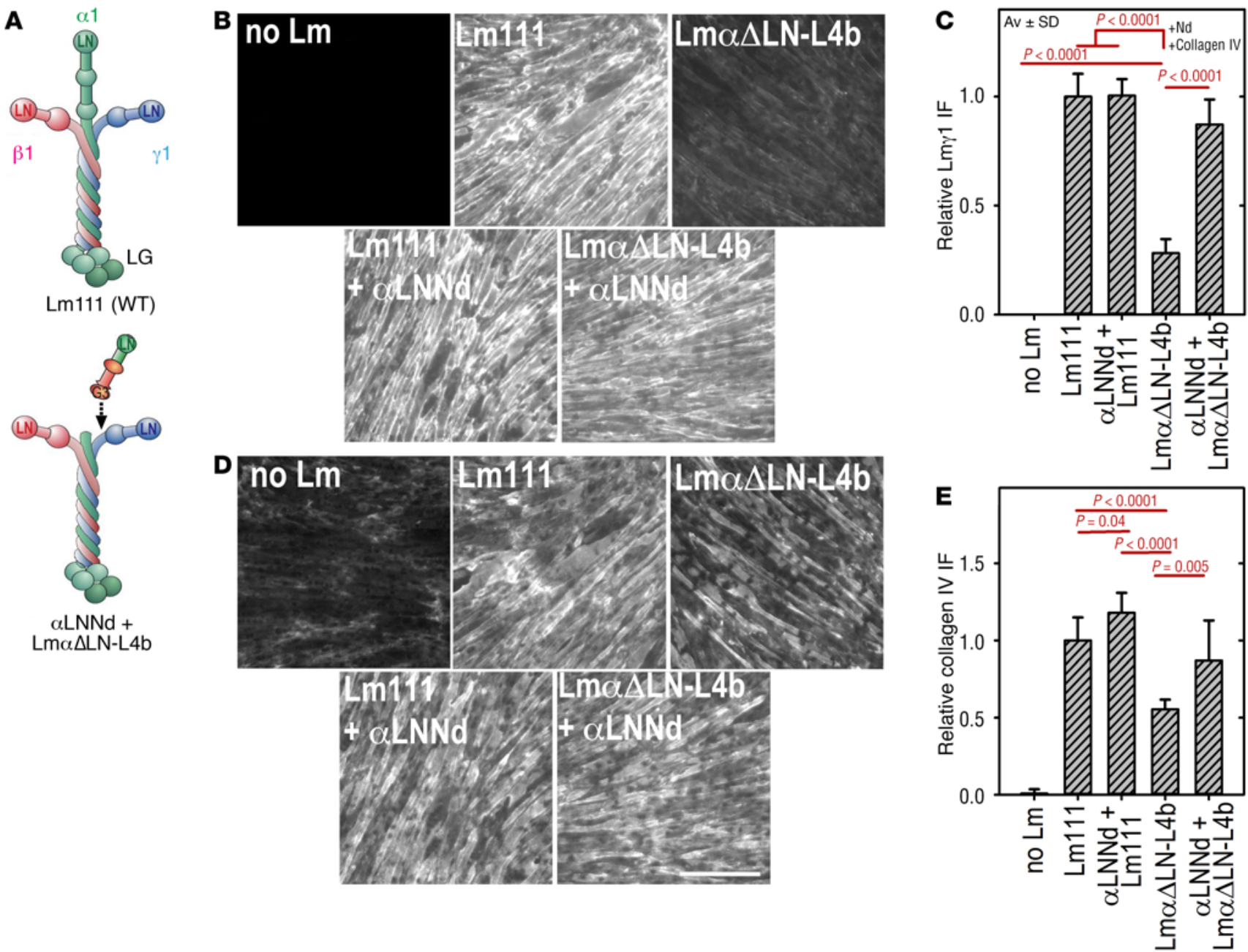

Figure 7. Effect of $\alpha \mathrm{LNNd}$ on nonpolymerizing laminin assembly on C2C12 myotubes. (A) Lm111, in which the $\alpha 1$-short arm has been deleted (Lm $\alpha \Delta \mathrm{LN}$ L4b), binds to aLNNd. (B-E) Lawns of fused C2C12 myotubes were incubated with the indicated WT and polymerization-deficient Lm111 (28 $\mathrm{nM}$ ) in the presence of nidogen-1 ( $28 \mathrm{nM}$ ) and collagen IV $(14 \mathrm{~nm})$, without or with $\alpha \mathrm{LNNd}(28 \mathrm{nM})$ for 1 hour, followed by washing, fixation, and immunostaining. Representative myotube images are shown in B (Lm $\gamma 1$ immunofluorescence) and D (collagen IV immunofluorescence) (scale bar: $200 \mu \mathrm{m}$ ), with corresponding graphs of the average summed intensities \pm SD $(n=7-9 \times 10$ fields/condition; 1-way ANOVA with pairwise Holm-Sidak comparisons) shown in C and $\mathbf{E}$. A substantial decrease of nonpolymerizing Lm $\alpha \Delta \mathrm{LN}-\mathrm{L} 4 \mathrm{~b}$ accumulation compared with WT control was prevented when $\alpha \mathrm{LNNd}$ was coincubated with $\mathrm{Lm} \alpha \Delta \mathrm{LN}-\mathrm{L} 4 \mathrm{~b}$. In contrast, $\alpha \mathrm{LNNd}$ did not alter WT laminin accumulation. Smaller collagen IV differences were detected among polymerizing and nonpolymerizing laminins. IF, immunofluorescence; Lm, laminin.

The polymerization-dependent decrease of laminin accumulation observed in vitro was also observed in $d y 2 J$ muscle, as detected in immunostained tissue and measured in extracts. Transgene-driven $\alpha \mathrm{LNNd}$ expression acted as an effective enabler of laminin accumulation in dystrophic muscle. This increase was accompanied by substantial improvements in muscle strength and muscle histology. Our evaluation of $d y 2 J$ laminin occupancy by $\alpha$ LNNd suggests that approximately half of the nonpolymerizing laminin is bound to endogenous nidogen-1, a major BM component of muscle and other tissues. This finding is not surprising, given that $\alpha$ LNNd must compete with endogenous nidogen- 1 for binding to laminins and that nidogen-1 is normally in molar excess to laminin. The important lesson may be that total $\alpha \mathrm{LNNd}$ occupancy is not essential for a major phenotypic improvement.

A direct approach of restoring laminin expression by germline transgene expression of the entire $L m \alpha 1$ subunit was very effective in its ability to restore normal function in dystrophic Lama2-KO mice $(33,34)$, an approach that may also benefit the $d y 2 J$ phenotype if the induced expression of the full-length laminin $\alpha$-subunit is high enough to compete with the defective endogenous Lma2 subunit for heterotrimeric chain assembly. However, application of this therapeutic approach will be difficult to translate to patients, given the large size (9.3 kb) of the DNA construct. Whether recombinant protein injection would be efficacious remains to be established (44). Adeno-associated virus (AAV) is one of the most promising of the gene delivery systems, as high expression levels can be achieved. However, the DNA-coding insert, inclusive of its promoter, is limited to approximately $5 \mathrm{~kb}$ (45). Transgene- and AAV-mediated expression has been found to similarly improve the severe laminindeficient dystrophy of the $d y^{W} / d y^{W}$ mouse, likely acting by increasing Lm411 receptor binding $(22,46)$. The current study suggests that AAV expression of $\alpha$ LNNd would be beneficial for the treatment of laminin deficiency for those patients with a defective LN domain and 
A

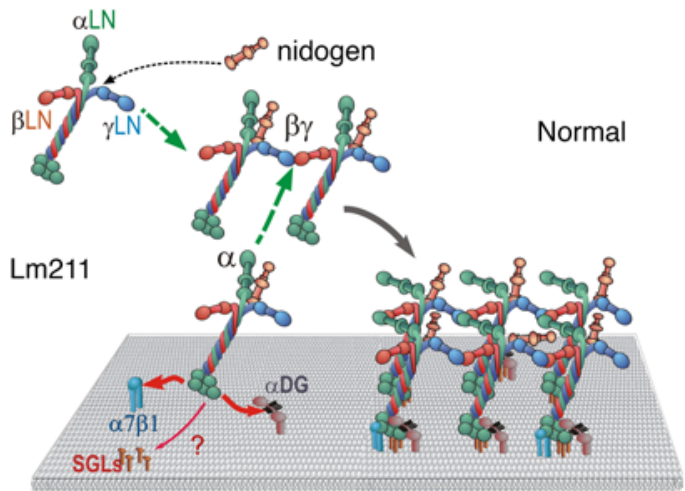

B
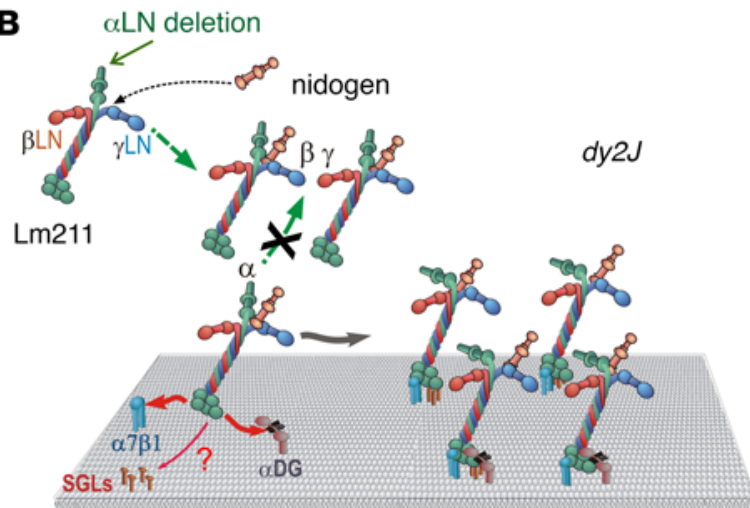

C

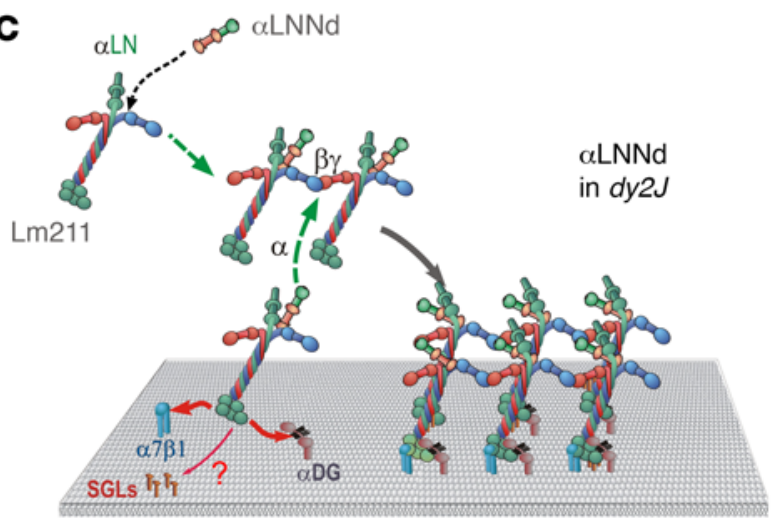

Figure 8. Model of laminin assembly in normal, dystrophic, and $\alpha \mathrm{LN}$ Nd-repaired muscle. (A) Normally, Lm211 adhesion (LG domain-mediated) to sarcolemmal $\alpha \mathrm{DC}$ and $\alpha 7 \beta 1$ integrin is coordinated with its polymerization. Assuming similarity with Schwann cell BM assembly, sulfated glycolipids (SGLs) might contribute to laminin adhesion in muscle as well. The heparan sulfate chains of perlecan and muscle agrin are thought to mediate bonds between laminin and nidogen and the collagen network. (B) In the dy2J (and some cases of human Lma2) dystrophic state, Lm211 can still adhere to the sarcolemma but has lost its ability to polymerize. Findings of this study suggest that secreted laminin accumulates at reduced levels in the BM because of the loss of binding cooperativity with other architectural and receptor components. (C) In the Tg+dy2J mouse, $\alpha \mathrm{LNNd}$ binds to the defective nonpolymerizing $\operatorname{Lm} \alpha 2$, thereby enabling self-assembly. Endogenous nidogen-1 competes with $\alpha$ LNNd for binding to laminins, creating a mixture of polymerized and nonpolymerized laminins, the former favored for assembly into stable BM. While Lm411 polymerization is also enabled by $\alpha$ LNNd, the small increase is largely confined to capillaries adjacent to the myofibers in dy2J muscle. that, in combination with mini-agrin (mA), this expression might be beneficial for all laminin-deficient dystrophies.

In summary, the current study demonstrates that repair of a laminin polymerization defect can substantially ameliorate a laminin LN-deficient muscular dystrophy, affecting BM assembly, muscle histology, and muscle strength. It also illustrates the value of an in vitro model for BM assembly to predict outcomes in vivo. The key interactions identified in this study are that the laminin must polymerize, must bind to its receptors, and must bind to nidogen to form an effective bridge to collagen IV. These interactions create a stable BM that supports muscle function (Figure 8). If a laminin polymerization domain is lost, Lm211 is rarified in the BM, thereby destabilizing it. This deficit can be repaired with $\alpha \mathrm{LNNd}$, a laminin-binding protein modified to replace the defective domain and interact with the defective laminin to assemble a stable BM. Finally, we believe our study sets the stage for the development of therapeutic approaches utilizing engineered laminin-binding proteins that provide missing functions.

\section{Methods}

DNA constructs. Expression vectors for the mouse laminin $\alpha 1$, human $\beta 1$, and human $\gamma 1$-subunits; for deletions of $\alpha 1 \mathrm{LN}, \alpha 1 \mathrm{LN}-\mathrm{L} 4 \mathrm{~b}, \alpha 1 \mathrm{LG1}-$ $5, \beta 1 \mathrm{LN}$, and $\gamma 1 \mathrm{LN}$ for $\alpha \mathrm{LNNd}$; for mouse nidogen-1; and for chick non-neural $\mathrm{mA}$ were generated as previously described $(14,15)$. To generate the $\alpha \mathrm{LNNd}$ expression vector for the transgenic mouse line, the LNNd ORF from the pcDNA3.1 Zeo plasmid was digested with $\mathrm{XbaI}$ and XhoI and inserted into the muscle-specific expression vector BS mck+polyA (22), which contains the muscle-specific creatine kinase promoter MCK (Figure 1A). The BS mck+polyA vector was also digested with the same enzymes to insert the $\alpha \mathrm{LNNd}$ ORF into the multiple cloning sites of the expression vector. DNA was linearized with restriction endonuclease PvuI for DNA injections.

A cDNA construct to express mouse laminin- $\alpha 4$ with an $\mathrm{N}$-terminal FLAG tag was prepared with a KpnI and NotI digest of $\alpha 4$ Blunt II TOPO (GE Dharmacon; EMM1002-97544805) and ligation into pcDNA3.1 puro. An N-FLAG tag was added into an NheI-BstEII site with overlapping PCR (1 forward, 5'-TGGCTGACCGCCCAACGAC-3'; 1 reverse, 5'-CAGGTGGGGCAAGGGACAGG-3'; 2 forward, 5'-GACGACGATGACAAGGCGTCAGGGGACGGCAAT-3'; 2 reverse, 5'-ATTTGCCGTCCCCTGACGCCTGTCATCGTCGTC- $3^{\prime}$ ). Mouse $\alpha 5$ (a gift of Jeff Miner, Washington University School of Medicine, St. Louis, MO, USA) was inserted into pcDNA3.1 puro with EcoRI as described previously (47). A FLAG tag sequence was added to the C-terminus with overlapping PCR (1 forward, 5'-GCCTGTATGTGGATGACCAGCTAC-3'; 1 reverse, 5'-CGACGATGCAGTTCAATGGTCG-3'; 2 forward, 5'-CATGACTACAAGGACGACGATGACAAG-3'; 2 reverse, 5'-GTCCTTGTAGTCATGCCAAAGTAGCGG-3') at an NsiI site. The human $\gamma 1$ ORF was moved from pRC-Cmv2 to pcDNA3.1neo with BamHI.

Recombinant and native laminins and other proteins. HEK293 cell lines stably expressing recombinant Lm111 heterotrimers (WT and deletion modified) and $\alpha$ LNNd were purified by HA-affinity chromatography as described previously in detail (36). Lm211 from cells grown in the presence of the furin inhibitor 1 ( $5 \mu \mathrm{M}$ in medium; Calbiochem; catalog 344930) to reduce LG domain cleavage (48) was purified by heparin affinity chromatography (heparin-agarose column), keeping the fraction that eluted between 250 and $500 \mathrm{mM} \mathrm{NaCl}$ in $50 \mathrm{mM}$ Tris-HCl, pH 7.4. HEK293 cells stably expressing Lm411 or Lm511 with an Lma 4 N-terminal FLAG tag or an Lma5 C-terminal FLAG tag were 
prepared using FLAG-M2 Ab affinity chromatography (14). Recombinant mouse nidogen-1 was purified from conditioned medium using HisPur-cobalt chelating chromatography (Thermo Fisher Scientific; catalog 89965) and detected with a rabbit polyclonal Ab as previously described (15) or with anti-entactin (Chemicon; catalog MAB1946). Type IV collagen was extracted from lathyritic mouse EngelbrethHolm-Swarm (EHS) tumor and purified as previously described (37).

Protein determinations. Molar laminin concentrations were determined by densitometry of Coomassie blue-stained acrylamide gels compared with an EHS-laminin (710-kDa protein mass) standard (19), corrected for changes in the calculated mass (Lm $\Delta \alpha \mathrm{LN}-\mathrm{L} 4 \mathrm{~b}, 558 \mathrm{kDa})$, Lm-411 (513 kDa), Lm-511 (784 kDa), and Lm-211 (718 kDa) as previously described $(14,15)$. Absorbance at $280 \mathrm{~nm}$ was used to measure the concentration of $\alpha \mathrm{LNNd}(157 \mathrm{kDa})$.

Culturing, immunostaining, and analysis of BM assembly in C2C12 myotubes. Mouse C2C12 myoblasts (ATCC CRL-1772) were maintained and differentiated into myotubes on using a previously described method (49). Myoblasts were maintained in DMEM, 10\% FCS, and penicillin-streptomycin. Cells between passages 6 and 7 were plated onto 24 -well dishes at 250,000 cells per well and incubated at $37^{\circ} \mathrm{C}$ overnight. The following day, the media were changed to DMEM, $5 \%$ horse serum (Gibco, Thermo Fisher Scientific), and 1\% penicillinstreptomycin, and cells were allowed to fuse for 4 to 5 days at $37^{\circ} \mathrm{C}$. Myotube cultures were washed 3 times with PBS, followed by fixation in $3.5 \%$ paraformaldehyde in PBS for 30 minutes at room temperature (RT). Cultures were blocked overnight at $4^{\circ} \mathrm{C}$ with $5 \%$ goat serum and $0.5 \%$ BSA in PBS. Myotube lawns were stained with primary polyclonal and monoclonal Abs specific for laminin subunits, laminin domains, nidogen- 1 , collagen IV, $\alpha \mathrm{LNNd}, \alpha \mathrm{DG}$, and integrin- $\beta 1$ (see Supplemental Table 1). Detection of bound primary Abs was accomplished with Alexa Fluor 488 and 647 goat anti-rabbit, anti-chicken, and antimouse IgG secondary Abs (Molecular Probes) at 1:200 and FITCconjugateddonkeyanti-mouse IgMat1:100 (JacksonImmunoResearch) with nuclear counterstaining with 4',6-diamidino-2-phenylindole (50). Myofiber cultures were viewed by indirect immunofluorescence using an inverted microscope (IX70; Olympus) fitted with an IXFLA fluorescence attachment and a MicroMax 5-mHz CCD camera (Princeton Instruments) controlled by IP Lab 3.7 (Scanalytics). Digital images were recorded (5-12 fields, each 1,300 × 1,030 pixels) using a $\times 10$ objective microscope objective, with the same exposure time for a given primary and secondary $\mathrm{Ab}$ set when comparisons were to be made. Fluorescence levels were estimated from the digital images with ImageJ software (NIH), with calculations performed in Microsoft Excel. A protocol previously described for the measurement of laminin on Schwann cells (14) was modified, such that the average background/pixel was determined from the fields of untreated myotube lawns by automatic mean-threshold segmentation. The overall background was calculated as the background/pixel multiplied by the total area of myotube lawn occupying the field and subtracted from the sample fluorescence. Data are expressed as the mean \pm SD of normalized summed intensities in SigmaPlot 12.5 (Systat Software).

Mice. $D y^{2 J} /+$ mice (on a C57BL/6J background) were purchased from The Jackson Laboratory and used for breeding. For genotyping, genomic DNA was purified from mouse tail clippings obtained at the time of weaning in $0.1 \mathrm{M} \mathrm{NaOH}$ buffer and boiled at $100^{\circ} \mathrm{C}$ in PCR tubes for 15 minutes. Samples were cooled, vortexed, and diluted into $40 \mathrm{mM}$ Tris buffer, $\mathrm{pH}$ 8.0. Genotyping PCRs were performed with $1 \mu$ l genom- ic DNA per 20- $\mu$ l reaction according to the Jumpstart Taq instructions (Sigma-Aldrich; catalog P2893). PCRs were performed for $d y 2 J$ mice with primers at final concentrations of $5 \mathrm{pmol} / \mu \mathrm{l}$ using $d y^{2 J}$ forward, $5^{\prime}$-TCCTGCTGTCCtGAATCTTG- $3^{\prime}$ and $d y^{25}$ reverse, $5^{\prime}$-CATTCTGTGCCAGGGAGTC-3' to generate a 300 -bp product under the following thermocycler conditions: $94^{\circ} \mathrm{C}$ for 5 minutes and 35 cycles of $94^{\circ} \mathrm{C}$ for 30 seconds; $52^{\circ} \mathrm{C}$ for 30 seconds; $72^{\circ} \mathrm{C}$ for 1 minute; and ending with $72^{\circ} \mathrm{C}$ for 3 minutes. Following amplification, the PCR products were digested for 2 hours at $37^{\circ} \mathrm{C}$ with Fermentas Fast Digest NdeI Enzyme to yield either a 300-bp, 200-bp plus 100-bp, or 100-bp banding pattern when run on a $1 \%$ agarose gel to indicate the WT, $d y^{2 J} /+$, or $d y^{2 J} / d y^{2 J}$ genotypes, respectively (51). For transgenic mice, the BS-MCK/ $\alpha \mathrm{LNNd}$ expression construct was injected into $\mathrm{C} 57 \mathrm{Bl} / 6 \mathrm{~J}$ mouse oocytes at the Transgenic Mouse Core Facility of the University of Basel. Transgenic lines $(\mathrm{C} 57 \mathrm{Bl} / 6 \mathrm{~J})$ were identified by PCR and Southern blot analysis. Of the $2 \alpha$ LNNd-transgenic mouse lines generated, the line with higher detectable protein levels was used for the study. These mice were bred with $d y^{2 J} /+$ mice to generate dystrophic animals $\left(d y^{2 J} / d y^{2 J}\right.$ with or without $\alpha \mathrm{LNNd}$ ) and controls (+/+ and $d y^{2 J} /+$ with or without $\left.\alpha \mathrm{LNNd}\right)$. No histological defects, gait abnormalities, or weakness was detected among the control genotypes.

Tissue harvesting and mouse muscle histology. Forelimbs and hind limbs were dissected from euthanized mice at 3 to 4 and 11 weeks of age. For paraffin embedding, forelimbs and hind limbs were fixed in $10 \%$ buffered formalin overnight (Sigma-Aldrich; catalog SF93-4) after removal of skin. Specimens were incubated overnight at RT with an acidic decalcification solution (Cal-Ex CS510-1D; Thermo Fisher Scientific) and washed in running tap water for several hours, refixed in $10 \%$ formalin overnight, rinsed, cross sectioned, and maintained in $70 \%$ ethanol for tissue processing. Tissues were embedded, sectioned at a thickness of $5 \mu \mathrm{m}$, and stained with $\mathrm{H} \& \mathrm{E}$ at the Histopathology Core (The Rutgers Cancer Institute of New Jersey, New Brunswick, New Jersey, USA). Slide-mounted, unstained sections cut adjacent to those stained with H\&E were deparaffinized and stained with Picrosirius red in picric acid to detect collagens (Sirius Red Stain Kit, NovaUltra, IW-3012; IHC World) according to the manufacturer's instructions. Composite images of extensor carpi radialis and rectus femoris were generated from partially overlapping images. Collagen levels were estimated by determining the red fluorescence of entire muscle cross sections from different mice stained in parallel (52). A segmentation range was defined and applied to all mice evaluated to capture the fibrosis accumulation within the given muscle using IPLab 3.7 software (Scanalytics Inc.). That segmentation value (sum value in pixels) was divided by the area (in pixels) of the muscle group to achieve the percentage of fibrosis for the rectus femoris muscle for a given mouse. For frozen sections, unfixed limbs were cross sectioned, mounted, and oriented in OCT and rapidly frozen in liquid nitrogen. For electron microscopic analysis, skeletal muscle was fixed in $0.5 \%$ gluteraldehyde and $0.2 \%$ tannic acid in PBS for 1 hour, washed with 0.1 M sodium cacodylate buffer, transferred to modified Karnovsky's fixative, post-fixed in $1 \%$ osmium tetroxide for 1 hour, and prepared and imaged as previously described (36).

Morphometry. Myofiber cross-sectional areas and perimeter lengths were measured from bright-field images of paraffin-embedded, stained sections and immunostained frozen sections. Images ( $\times 20$ objective) from paraffin-embedded sections were recorded with a Nikon Eclipse 50i microscope fitted with a Diagnostic model 18.2 color mosaic camera 
under the control of SPOT software (version 4.6.4.5; Diagnostic Instruments, Inc). Composite images of whole muscle in cross sections were assembled in Adobe Photoshop CS6 from overlapping recorded images. Individual myofiber perimeters of a given muscle were traced on a Wacom Cintiq 22HD screen in ImageJ, with conversion to myofiber areas. The fraction of myofibers with central nuclei in the muscle was used to estimate the degree of myofiber regeneration.

Abs. Abs against the Lm $\alpha 1$ domains of $\alpha \mathrm{LNNd}$, laminin subunits $\alpha 2, \alpha 4, \alpha 5, \beta 1 / 1$, and $\gamma 1$, nidogen- 1 , nidogen domains $\mathrm{G} 2-\mathrm{G} 3$ common to $\alpha \mathrm{LNNd}$ and nidogen-1, collagen IV, $\alpha \mathrm{DG}$, and integrin $\beta 1$ are described in Supplemental Table 1. Generally, rabbit and chicken polyclonal antisera were affinity purified on their respective ligands by affinity chromatography and cross absorption. Columns of 3 to $4 \mathrm{ml}$ of sepharose $4 \mathrm{~B}$ coupled to ligands were prepared using cyanogen bromide-activated beads at 1 to $2 \mathrm{mg} / \mathrm{ml}$ ligand/ml beads. Loaded samples were eluted with $0.1 \mathrm{M}$ acetic acid, $\mathrm{pH}$ adjusted to neutrality, and dialyzed into PBS. For cross absorption, the Ab was passed through the column in PBS with retention of the unbound fraction. Cross absorption was repeated until adequate specificity could be demonstrated by ELISA and Western immunoblotting (protein in muscle lysates and recombinant Lm111, Lm211, Lm411, and Lm511).

Tissue preparation, Ab staining, and microscopy. Skeletal muscles were embedded in OCT (Tissue-Tek), flash frozen in liquid nitrogen, and stored at $-80^{\circ} \mathrm{C}$. For sectioning, frozen blocks were first allowed to equilibrate to $-20^{\circ} \mathrm{C}$ overnight. Five-micron-thick sections were cut with a cryostat (Leica CM 1850) at $-20^{\circ} \mathrm{C}$ and adhered to positively charged slides (Thermo Fisher Scientific). Sections were then washed for 5 minutes in TBS-50 (or 30 minutes in TBS-50 containing 0.1\% NP40 for detection of $\alpha \mathrm{DG}$ and integrin), followed by fixation in $3.2 \%$ paraformaldehyde in PBS for 15 minutes at RT. Slides were washed in PBS and blocked in $5 \%$ goat serum overnight at $4^{\circ} \mathrm{C}$. Primary Abs were added the following day for 1 to 3 hours at RT and washed 3 times in PBS for 10 minutes. Secondary Abs conjugated with fluorescent probes were added for 1 hour at RT, followed by 30 minutes of 3 PBS washes of 10 minutes each. Slides were mounted with coverslips in 6\% DABCO (1,4-diazabicyclo [2.2.2]octane) in glycerol. Detection of bound primary Abs in fixed, frozen sections was accomplished with Alexa Fluor 488 and 647 goat anti-rabbit, chicken, and mouse IgG secondary Abs (Molecular Probes) at 1:200. Tissue sections were stained simultaneously and Ab titers were measured to ensure linear detection of BM components. Regions of muscle were matched between genotypes, and the same exposure times and normalizations were applied to all images being compared.

In order to estimate and compare the immunofluorescent intensities for different laminin subunits, multiple near-adjacent $\times 20$ fields of cross sections from triceps brachii muscle were recorded and subjected to image segmentation analysis in ImageJ. A mean threshold signal value was determined to correspond to the BM zone of the image, followed by collection of area and intensity density (sum) values. A mean background/pixel value for the non-BM zones was also determined by the same method, multiplied by the number of BM pixels, and subtracted from the BM summed values. The average and SD were then calculated. BM lengths were then determined and summed for each image by tracing the BMs with a stylus on a Wacom tablet in ImageJ. The summed intensities were divided by the BM lengths, with calculation of the average and SD of 8 to 12 images per condition.

Extraction and analysis of laminins from muscle. Neutral salt buffer containing NP40 (buffer fraction, B) was used to extract intracellular laminins and free extracellular laminins. Bacterial collagenase, which disrupts collagen bonds that retain laminins within the $\mathrm{BM}$, was used to release laminins (collagenase fraction, $\mathrm{C}$ ) from matrix in a manner previously observed with EHS matrix, placenta, and muscle, (18, 20, 25). EDTA, a chelating agent that inhibits laminin polymerization, integrin binding, and dystroglycan binding, was used to extract laminin, now released from its collagen bonds from matrix (EDTA fraction, E). SDS, which breaks nearly all noncovalent bonds, was then used to extract residual laminin (SDS fraction, $\mathrm{S}$ ) that might be more tightly embedded in the BM matrix. These 3 sequential extractions (C plus $\mathrm{E}$ plus S) were combined to form the BM matrix fraction after removal of the soluble/ intracellular laminins released by buffer. Mouse skeletal muscle fragments of equal mass were homogenized (Polytron) in $2 \mathrm{ml}$ of ice-cold TBS-50 (50 mM Tris, $90 \mathrm{mM} \mathrm{NaCl}, \mathrm{pH}$ 7.4) with protease inhibitors (Sigma-Aldrich; catalog P8340), then incubated on ice for 30 minutes with 1\% NP40. Homogenates were transferred to 2-ml Eppendorf tubes and centrifuged $\left(15,800 \mathrm{~g}, 20 \mathrm{~min}, 4^{\circ} \mathrm{C}\right)$. The supernatants (buffer fraction) were saved, and the pellets were suspended in a collagenase solution $(0.2 \mu \mathrm{g} / \mathrm{ml}$ bacterial collagenase [CLSPA; Worthington Biochemical] in TBS-50, $4 \mathrm{mM}$ calcium chloride, and protease inhibitors), incubated at $37^{\circ} \mathrm{C}$ for 2 hours with frequent agitation, and centrifuged for 20 minutes with the supernatant saved (collagenase fraction). Pellets were then suspended in ice-cold EDTA buffer $(20 \mathrm{mM}$ EDTA in TBS-50 and protease inhibitors), rocked overnight at $4^{\circ} \mathrm{C}$, centrifuged (20 min, $4^{\circ} \mathrm{C}$ ), and the supernatants saved (EDTA fraction). The final pellet residue was then extracted with Laemmli SDS-solubilizing buffer and the supernatant (SDS fraction) saved after centrifugation. Aliquots of sequential extracts for each step were analyzed in Western immunoblots with GADPH as a loading control and $\alpha$-actinin as a myofiber control. Each fractional volume was recorded for later calculations.

ELISA sandwich assay to compare laminins in muscle extracts. Ninetysix-well high protein-binding Costar plates were coated $(1-2 \mu \mathrm{g} / \mathrm{ml})$ with rabbit polyclonal anti-Lm $\alpha 2(2 \mu \mathrm{g} / \mathrm{ml})$, goat anti-Lm $\alpha 4(1 \mu \mathrm{g} / \mathrm{ml})$, or chicken anti-Lm $\beta 1 \gamma 1(1 \mu \mathrm{g} / \mathrm{ml})$. The wells were then washed and blocked with blocking buffer (PBS, 1\% BSA, 0.06\% Triton X-100). Twofold serial dilutions of muscle-extracted proteins were prepared in PBS containing $1 \%$ BSA and $1 \% \mathrm{NP} 40$ and applied $(0.1 \mathrm{ml})$ to the Ab-coated wells. After incubation for 2 hours at RT, unbound protein was removed and the wells washed, and then the wells were incubated with either chicken anti-Lm $\beta 1 \gamma 1(1 \mu \mathrm{g} / \mathrm{ml}$ in each well) to detect Lm211 or Lm411, or (for matrix to increase sensitivity) goat anti-Lma4 on the laminins bound to chicken anti-Lm $\beta 1 \gamma 1$. For detection of total $\beta 1 \gamma 1$-laminins, wells were coated with rabbit anti-nidogen G2-G3 domains (common to nidogen and $\alpha \mathrm{LNNd}$ ) and detected with chicken anti-Lm $\beta 1 \gamma 1$. After washing, wells were incubated with anti-chicken IgY or anti-goat IgG coupled to HRP $(1: 5,000)$, washed again, and then incubated with TMB $\left(3,3^{\prime}, 5,5^{\prime}\right.$-tetramethylbenzidine) color reagent for 3 minutes. Absorbance was measured at A450 following the addition of sulfuric acid to halt further color development. The use of serial dilutions for unknowns rather than same-dilution replicates allowed for determination of whether the unknowns fell into the sensitive, near-linear range of the assay. Averages were determined for the first 4 dilutions (ratio of A450/lysate volumes) and divided by the corresponding mean control values. The normalized values for 4 different mice per condition were then averaged and plotted. These values were divided by the $\alpha$-actinin levels for each mouse to correct for differences in myofiber content (determined as averaged and normalized intensity levels from 3 repeat immunoblots). 
Immunoprecipitation and immunoblot analysis. Equal fractions of combined lysates were pre-cleared with either protein-A agarose beads (Sigma-Aldrich) for 30 minutes (for rabbit Ab immunoprecipitations) or goat anti-chicken IgY agarose beads (GeneScript) for 30 minutes (for chicken Ab immunoprecipitations). The lysates were then immunoprecipitated with Abs against either $\alpha$ LNNd (chicken anti-F2, $5 \mu \mathrm{g} / \mathrm{ml}$ ) or rabbit anti-nidogen (G2-G3 domains, $5 \mu \mathrm{g} / \mathrm{ml}$ ). Protein A agarose beads (for Lm $\beta 1, \gamma 1$-, and $\alpha 2$-subunits) or anti-chickenconjugated agarose beads (for $\alpha$ LNNd) were added and incubated for 1 hour with gentle agitation. The beads were removed by centrifugation (10 s) and the supernatants collected. Beads were then washed 3 times with cold IP-wash buffer (50 mM Tris, $90 \mathrm{mM} \mathrm{NaCl}, 1 \mathrm{mM}$ EDTA, $0.06 \%$ Triton X-100). Laemmli buffer containing mercaptoethanol was added to the pellets. After boiling for 5 minutes, supernatant aliquots were loaded onto a 7.5\% SDS-PAGE reducing gel and electrophoresed (80 min, 120 volts). Gel-embedded proteins were transferred onto PVDF membranes in cold transfer buffer $(25 \mathrm{mM}$ Tris base, $190 \mathrm{mM}$ glycine, $0.1 \%$ SDS, $20 \%$ methanol, $\mathrm{pH} 8.3$ ) at 100 volts for 1 hour. Membranes were blocked overnight in $5 \%$ milk protein (50 $\mathrm{mM}$ Tris, $150 \mathrm{mM} \mathrm{NaCl}, 0.2 \%$ Tween-20). Primary Ab against either laminin- $\beta 1 / \gamma 1(1 \mu \mathrm{g} / \mathrm{ml})$ or nidogen $(1 \mu \mathrm{g} / \mathrm{ml})$ was added to Western Ab buffer (50 mM Tris, $150 \mathrm{mM} \mathrm{NaCl}, 1 \%$ BSA, 0.2\% Tween-20) for 3 hours at RT, followed by three 10-minute washes with Western wash buffer (50 mM Tris, $150 \mathrm{mM} \mathrm{NaCl}, 0.2 \%$ Tween-20). Secondary Ab (goat anti-rabbit horse-radish peroxidase conjugated) was added at 1:3,000 to Western Ab buffer for 1 hour at RT, with gentle agitation. Membranes were washed 3 times (10 min each) and exposed simultaneously with equal volumes of West Femto Kit components (Enhancer Solution plus Peroxide Buffer; Thermo Fisher Scientific) on the BioRad Gel-Dock System and evaluated by Quantity One software (BioRad). Relative intensities of $\mathrm{Lm}-\beta 1 / \gamma 1$ bands were determined using GelAnalyzer software (2010a; Lazar Software).

qRT-PCR analysis. RNA was isolated and stabilized from tissues with reagents provided by an RNeasy Plus Mini Kit (QIAGEN). qRT-PCR was performed to quantify laminins, integrins, and dystroglycan, and fibronectin relative to the housekeeping GAPDH gene using a StepOnePlus System (Applied Biosystem) as described previously (53).

Grip strength measurements. Grip strength was measured with a mouse Grip Strength Meter (Columbus Instruments) according to the manufacturer's instructions (User Manual 0167-007). Forelimb measurements were performed with the wire triangle attachment (pulling the mouse rearward by the tail), while all-limb and hind limb measurements were performed with the angled grid attachment, pulling the mouse towards the meter by the tail after engagement of all limbs, or pulling the mouse by the tail by only the hind limbs (forelimbs positioned above the mesh by holding the mouse by the scruff of the neck). The recommended 5 consecutive measurements per mouse protocol was increased to 10, with averaging of the 6 (rather than 3 ) highest values. Data are expressed as grams of peak force divided by the mouse's weight.

Statistics. Averages and SD were calculated from measured values obtained from 3 or more images, and morphometric measurements of perimeters and areas were performed using the statistical package in
SigmaPlot 12.5 (Systat Software) or Microsoft Excel. Averages and SEM were determined from the mean values of consecutive sets of determinations (e.g., grip strength) from different mice and from the mean values of myofiber cross-sectional areas from different mice. Three or more conditions were compared by one-way ANOVA followed by Holm-Sidak pairwise analysis in SigmaPlot, while two conditions were evaluated by a two-tailed $t$ test using Microsoft Excel. A difference was considered significant for $P$ values of 0.05 or less and trended toward significance for $P$ values of greater than 0.05 but less than or equal to 0.10 .

Study approval. The mouse protocol (112-074) for the study was approved by the IACUC committee of Rutgers University and Robert Wood Johnson Medical School. The biosafety protocol (IBC no. 13-574) for the study was approved by the Institutional Biosafety Committee of Rutgers University.

\section{Author contributions}

KKM prepared constructs, expressed and characterized recombinant proteins, characterized Abs, conducted myotube experiments, analyzed mouse tissues, prepared images and statistical analysis of immunostained images, and was responsible for $\mathrm{Ab}$ and biochemical experiments. SCC carried out initial evaluations with myotubes and mice, including breeding, genotyping, protein characterizations, and qualitative evaluations of muscle histology. SM supervised the generation of the transgenic mice and characterized expression of the transgenes. JRR performed mouse screen immunoblots. MAR oversaw the generation and characterization of the transgenic mouse and provided advice on analysis as part of an ongoing collaboration to study the role of laminin-binding proteins in muscular dystrophy. PDY coordinated the study, performed grip strength measurements, morphometric analysis, data calculations, mouse breeding and analysis, and electron microscopy and wrote the manuscript.

\section{Acknowledgments}

This study was supported by NIH grant R01-DK36425 (to PDY) and grants from the Cantons of Basel-Stadt, Basel-Landschaft, Swiss Foundation for Research on Muscle Diseases, Association Française contre les Myopathies, and Neuromuscular Research Association Basel (to MAR). "We thank Julianne Avrutik and Samantha Hobbs (Robert Wood Johnson Medical School, Piscataway, NJ, USA) for assisting in the analysis and Robert Vasson (Robert Wood Johnson Medical School) for developing a script for Image J to rapidly determine summed intensities for given segmentation ranges. We thank Rajesh Patel (Electron Microscopy Services, Robert Wood Johnson Medical School) for preparing samples for electron microscopy.

Address correspondence to: Peter D. Yurchenco, Department of Pathology and Laboratory Medicine, Rutgers - Robert Wood Johnson Medical School, 675 Hoes Lane West, Piscataway, New Jersey 08854, USA. Phone: 732.235.5166/4674; E-mail: yurchenc@ rwjms.rutgers.edu.
1. Jimenez-Mallebrera C, Brown SC, Sewry CA, Muntoni F. Congenital muscular dystrophy: molecular and cellular aspects. Cell Mol Life Sci. 2005;62(7-8):809-823.
2. Allamand V, et al. Mild congenital muscular dystrophy in two patients with an internally deleted laminin alpha2-chain. Hum Mol Genet. 1997;6(5):747-752.
3. Gavassini BF, et al. Clinical and molecular characterization of limb-girdle muscular dystrophy due to LAMA2 mutations. Muscle Nerve. 2011;44(5):703-709. 
4. Yurchenco PD. Integrating Activities of Laminins that Drive Basement Membrane Assembly and Function. Curr Top Membr. 2015;76:1-30.

5. Hussain SA, Carafoli F, Hohenester E. Determinants of laminin polymerization revealed by the structure of the $\alpha 5$ chain amino-terminal region. EMBO Rep. 2011;12(3):276-282.

6. Bönnemann CG, et al. Diagnostic approach to the congenital muscular dystrophies. Neuromuscul Disord. 2014;24(4):289-311.

7. Chan SH, et al. Limb girdle muscular dystrophy due to LAMA2 mutations: diagnostic difficulties due to associated peripheral neuropathy. Neuromuscul Disord. 2014;24(8):677-683.

8. Colognato H, Yurchenco PD. Form and function: the laminin family of heterotrimers. Dev Dyn. 2000;218(2):213-234.

9. Brakebusch C, Fässler R. The integrin-actin connection, an eternal love affair. $E M B O J$. 2003;22(10):2324-2333.

10. Ervasti JM, Campbell KP. A role for the dystrophinglycoprotein complex as a transmembrane linker between laminin and actin. J Cell Biol. 1993;122(4):809-823.

11. Mayer U, et al. Absence of integrin alpha 7 causes a novel form of muscular dystrophy. Nat Genet. 1997;17(3):318-323.

12. Nishiuchi $R$, et al. Ligand-binding specificities of laminin-binding integrins: a comprehensive survey of laminin-integrin interactions using recombinant alpha3beta1, alpha6beta1, alpha7beta1 and alpha6beta4 integrins. Matrix Biol. 2006;25(3):189-197.

13. Han R, et al. Basal lamina strengthens cell membrane integrity via the laminin $G$ domain-binding motif of alpha-dystroglycan. Proc Natl Acad Sci U S A. 2009;106(31):12573-12579.

14. McKee KK, Harrison D, Capizzi S, Yurchenco PD. Role of laminin terminal globular domains in basement membrane assembly. J Biol Chem. 2007;282(29):21437-21447.

15. McKee KK, Capizzi S, Yurchenco PD. Scaffoldforming and Adhesive Contributions of Synthetic Laminin-binding Proteins to Basement Membrane Assembly. J Biol Chem. 2009;284(13):8984-8994.

16. Yurchenco PD, Patton BL. Developmental and pathogenic mechanisms of basement membrane assembly. Curr Pharm Des. 2009;15(12):1277-1294.

17. Yurchenco PD, Tsilibary EC, Charonis AS, Furthmayr H. Laminin polymerization in vitro. Evidence for a two-step assembly with domain specificity. J Biol Chem. 1985;260(12):7636-7644.

18. Yurchenco PD, Cheng YS, Colognato H. Laminin forms an independent network in basement membranes. J Cell Biol. 1992;117(5):1119-1133.

19. Yurchenco PD, Cheng YS. Self-assembly and calcium-binding sites in laminin. A three-arm interaction model. J Biol Chem. 1993;268(23):17286-17299.

20. Cheng YS, Champliaud MF, Burgeson RE, Marinkovich MP, Yurchenco PD. Self-assembly of laminin isoforms. J Biol Chem. 1997;272(50):31525-31532.

21. Garbe JH, Göhring W, Mann K, Timpl R, Sasaki T. Complete sequence, recombinant analysis and binding to laminins and sulphated ligands of the N-terminal domains of laminin alpha3B and alpha5 chains. Biochem J. 2002;362(Pt 1):213-221.

22. Moll J, et al. An agrin minigene rescues dystrophic symptoms in a mouse model for congenital muscular dystrophy. Nature. 2001;413(6853):302-307.

23. Talts JF, et al. Structural and functional analysis of the recombinant $G$ domain of the laminin alpha4 chain and its proteolytic processing in tissues. J Biol Chem. 2000;275(45):35192-35199.

24. Yu H, Talts JF. Beta1 integrin and alphadystroglycan binding sites are localized to different laminin-G-domain-like (LG) modules within the laminin alpha5 chain $G$ domain. Biochem J. 2003;371(Pt 2):289-299.

25. Colognato H, Yurchenco PD. The laminin alpha2 expressed by dystrophic dy (2J) mice is defective in its ability to form polymers. Curr Biol. 1999;9(22):1327-1330.

26. Yurchenco PD, Cheng YS, Campbell K, Li S. Loss of basement membrane, receptor and cytoskeletal lattices in a laminin-deficient muscular dystrophy. J Cell Sci. 2004;117(Pt 5):735-742.

27. Sunada Y, Bernier SM, Utani A, Yamada Y, Campbell KP. Identification of a novel mutant transcript of laminin alpha 2 chain gene responsible for muscular dystrophy and dysmyelination in dy2J mice. Hum Mol Genet. 1995;4(6):1055-1061.

28. Xu H, Wu XR, Wewer UM, Engvall E. Murine muscular dystrophy caused by a mutation in the laminin alpha 2 (Lama2) gene. Nat Genet. 1994;8(3):297-302.

29. Purvis A, Hohenester E. Laminin network formation studied by reconstitution of ternary nodes in solution. J Biol Chem. 2012;287(53):44270-44277.

30. Hopf M, Göhring W, Kohfeldt E, Yamada Y, Timpl R. Recombinant domain IV of perlecan binds to nidogens, laminin-nidogen complex, fibronectin, fibulin-2 and heparin. Eur J Biochem. 1999;259(3):917-925.

31. Sunada Y, Bernier SM, Kozak CA, Yamada Y, Campbell KP. Deficiency of merosin in dystrophic dy mice and genetic linkage of laminin M chain gene to dy locus. J Biol Chem. 1994;269(19):13729-13732.

32. Colognato H, Winkelmann DA, Yurchenco PD. Laminin polymerization induces a receptorcytoskeleton network. JCell Biol. 1999;145(3):619-631.

33. Gawlik K, Miyagoe-Suzuki Y, Ekblom P, Takeda S, Durbeej M. Laminin alpha1 chain reduces muscular dystrophy in laminin alpha2 chain deficient mice. Hum Mol Genet. 2004;13(16):1775-1784.

34. Gawlik KI, Li JY, Petersén A, Durbeej M. Laminin alpha1 chain improves laminin alpha2 chain deficient peripheral neuropathy. Hum Mol Genet. 2006;15(18):2690-2700.

35. Smirnov SP, Barzaghi P, McKee KK, Ruegg MA, Yurchenco PD. Conjugation of LG domains of agrins and perlecan to polymerizing laminin-2 promotes acetylcholine receptor clustering. J Biol Chem. 2005;280(50):41449-41457.

36. McKee KK, et al. Schwann cell myelination requires integration of laminin activities. J Cell Sci. 2012;125(Pt 19):4609-4619.

37. Yurchenco PD, Furthmayr H. Self-assembly of basement membrane collagen. Biochemistry. 1984;23(8):1839-1850.
38. Pöschl E, Fox JW, Block D, Mayer U, Timpl R. Two non-contiguous regions contribute to nidogen binding to a single EGF-like motif of the laminin gamma 1 chain. EMBO J. 1994;13(16):3741-3747.

39. Patton BL, et al. Distribution of ten laminin chains in dystrophic and regenerating muscles. Neuromuscul Disord. 1999;9(6-7):423-433.

40. Salmivirta K, Talts JF, Olsson M, Sasaki T, Timpl R, Ekblom P. Binding of mouse nidogen-2 to basement membrane components and cells and its expression in embryonic and adult tissues suggest complementary functions of the two nidogens. Exp Cell Res. 2002;279(2):188-201.

41. Yang D, et al. Coordinate control of axon defasciculation and myelination by laminin-2 and -8 . JCell Biol. 2005;168(4):655-666.

42. Chen ZL, Strickland S. Laminin gamma1 is critical for Schwann cell differentiation, axon myelination, and regeneration in the peripheral nerve. JCell Biol. 2003;163(4):889-899.

43. Yu WM, Feltri ML, Wrabetz L, Strickland S, Chen ZL. Schwann cell-specific ablation of laminin gamma1 causes apoptosis and prevents proliferation. J Neurosci. 2005;25(18):4463-4472.

44. Rooney JE, Knapp JR, Hodges BL, Wuebbles RD, Burkin DJ. Laminin-111 protein therapy reduces muscle pathology and improves viability of a mouse model of merosin-deficient congenital muscular dystrophy. Am J Pathol. 2012;180(4):1593-1602.

45. Grieger JC, Samulski RJ. Packaging capacity of adeno-associated virus serotypes: impact of larger genomes on infectivity and postentry steps. J Virol. 2005;79(15):9933-9944.

46. Qiao C, et al. Amelioration of laminin-alpha2deficient congenital muscular dystrophy by somatic gene transfer of miniagrin. Proc Natl Acad Sci USA. 2005;102(34):11999-12004.

47. Yazlovitskaya EM, et al. Integrin $\alpha 3 \beta 1$ regulates kidney collecting duct development via TRAF6-dependent K63-linked polyubiquitination of Akt. Mol Biol Cell. 2015;26(10):1857-1874.

48. Smirnov SP, McDearmon EL, Li S, Ervasti JM, Tryggvason K, Yurchenco PD. Contributions of the LG modules and furin processing to laminin-2 functions. J Biol Chem. 2002;277(21):18928-18937.

49. Kinose F, Wang SX, Kidambi US, Moncman CL, Winkelmann DA. Glycine 699 is pivotal for the motor activity of skeletal muscle myosin. J Cell Biol. 1996;134(4):895-909.

50. Li S, et al. Matrix assembly, regulation, and survival functions of laminin and its receptors in embryonic stem cell differentiation. J Cell Biol. 2002;157(7):1279-1290.

51. Vilquin JT, Vignier N, Tremblay JP, Engvall E, Schwartz K, Fiszman M. Identification of homozygous and heterozygous dy2J mice by PCR. Neuromuscul Disord. 2000;10(1):59-62.

52. Borges LF, Taboga SR, Gutierrez PS. Simultaneous observation of collagen and elastin in normal and pathological tissues: analysis of Sirius-red-stained sections by fluorescence microscopy. Cell Tissue Res. 2005;320(3):551-552.

53. Yang DH, et al. Renal collecting system growth and function depend upon embryonic $\gamma 1$ laminin expression. Development. 2011;138(20):4535-4544. 